\title{
A Growth Mixture Theory for Cartilage With Application to Cartilage Explants
}

Stephen M. Klisch ${ }^{1}$ Growth-Related Experiments on Silvia S. Chen Robert L. Sah

\author{
Department of Bioengineering, \\ University of California at San Diego, \\ La Jolla, CA \\ Anne Hoger \\ Department of Mechanical \\ and Aerospace Engineering, \\ University of California at San Diego, \\ La Jolla, CA
}

In this paper, we present a growth mixture model for cartilage. The main features of this model are illustrated in a simple equilibrium boundary-value problem that is chosen to illustrate how a mechanical theory of cartilage growth may be applied to growth-related experiments on cartilage explants. The cartilage growth mixture model describes the independent growth of the proteoglycan and collagen constituents due to volumetric mass deposition, which leads to the remodeling of the composition and the mechanical properties of the solid matrix. The model developed here also describes how the material constants of the collagen constituent depend on a scalar parameter that may change over time (e.g., crosslink density); this leads to a remodeling of the structural and mechanical properties of the collagen constituent. The equilibrium boundary-value problem that describes the changes observed in cartilage explants harvested at different stages of a growth or a degenerative process is formulated. This boundary-value problem is solved using existing experimental data for developing bovine cartilage explants harvested at three developmental stages. The solution of the boundary-value problem in conjunction with existing experimental data suggest the types of experimental studies that need to be conducted in the future to determine model parameters and to further refine the model. [DOI: 10.1115/1.1560144]

\section{Introduction}

Articular cartilage functions as a low friction, wear-resistant, load-bearing material $[1,2]$. Adult cartilage is composed of a relatively small fraction of cells, called chondrocytes, within a fluidfilled extracellular matrix. Chondrocytes are responsible for the turnover of matrix molecules, both in growth and in resorption. Two of the molecular components of the solid extracellular matrix (Fig. 1), proteoglycan and collagen, appear to be predominantly responsible for the functional mechanical properties of the tissue. The sulfated proteoglycan, aggrecan, consists of a protein core with attached glycosaminoglycan (GAG) chains. The GAG provides the tissue with a fixed negative charge that enhances the tissue's propensity to swell and to resist compressive loading $[3,4]$. The collagen is mostly present as fibrils immobilized in the tissue matrix by crosslinks $[5,6]$, forming a collagen network. The cross-linked collagen network resists the swelling tendency of the proteoglycan, and provides the tissue with tensile and shear stiffness and strength $[1,7,8]$.

Numerous studies have shown that the mechanical properties of cartilage are dependent on its composition. The aggregate modulus of adult cartilage has been positively correlated with GAG content [1] and, to a lesser extent, with collagen content $[9,10]$. The permeability of adult articular cartilage has been inversely related to GAG content measured as fixed charge density [11]. During fetal and postnatal development of bovine cartilage, there is little change in the GAG content [12-14] but there is an increase in both the collagen content and crosslink density $[15,16]$. These biochemical changes from fetal to adult cartilage are accompanied by an increase in the compressive and tensile moduli

${ }^{1}$ Address all correspondence to: Stephen M. Klisch, Ph.D., Assistant Professor, Mechanical Engineering Department, California Polytechnic State University, San Luis Obispo, CA 93407; Telephone (805) 756-1308; FAX (805) 756-1137 sklisch@calpoly.edu

Contributed by the Bioengineering Division for publication in the JOURNAL OF BIOMECHANICAL ENGINEERING. Manuscript received September 2001; revised manuscript received December 2002. Associate Editor: L. A. Taber. and each of these mechanical properties are significantly correlated with the GAG and/or the collagen content $[16,17]$.

Growth, resorption, and remodeling are fundamental processes that influence the size, shape, and properties of biological organs and tissues. Growth is "a normal process of increase in size of an organism as a result of accretion of tissue similar to that originally present [18]." Here, volumetric growth of a constituent is interpreted as the deposition of constituent mass that has the same mechanical properties as the existing material. Volumetric growth, at either the constituent or at the tissue level, may change the residual stress field (i.e., the stress field in the tissue or organ when all external loads have been removed) [19-21]. Since changes in the residual stress will alter the tissue's response to mechanical loads [22], volumetric growth leads to a change in the overall mechanical properties of the tissue. Resorption, "the loss of substance through physiologic or pathologic means [18]" is, roughly, the opposite of growth. On the other hand, to remodel is "to alter the structure of; remake [23]." Here, constituent remodeling is interpreted as a change in the structure of the constituent that alters the constituent's mechanical properties. Of course, such a change would have an impact on the overall mechanical properties of the tissue.

The cartilage growth mixture model developed here describes how the solid matrix remodels during cartilage growth due to two biological mechanisms: volumetric growth and remodeling of the proteoglycan and collagen constituents. When the constituents grow without remodeling, the composition of the solid matrix may evolve over time. Alternatively, when either constituent experiences remodeling without mass deposition or resorption, the properties of the solid matrix may evolve over time. The cartilage growth mixture model can describe how the change in composition caused by volumetric growth or, alternatively, the change in constituent remodeling causes the mechanical properties of solid matrix to evolve during a continuous growth process.

Due to the numerous parameters that influence the growth and mechanical properties of cartilage and the relative lack of experi- 


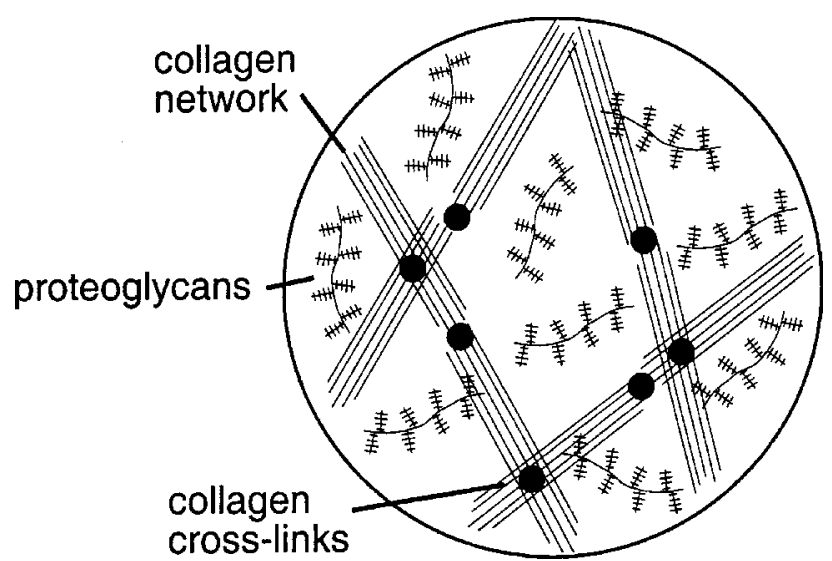

Fig. 1 Schematic of the major components of the solid extracellular matrix of articular cartilage.

mental data to determine these parameters at the present time, it is necessary to develop a model that, with several simplifying assumptions, can be used with existing data. In this paper, a continuum mechanics approach is used to develop a phenomological model of cartilage growth that may describe several of the salient features of cartilage growth. In the continuum approach, the primitive elements in the theory such as constituent densities, displacements, and stresses must be averaged over some reference volume. Thus, the continuum model of cartilage growth is intended to describe how these variables change in a volumeaveraged sense.

Continuum mixture theories [24-27] have been used to describe the mechanical behavior of articular cartilage $[28,29]$. However, none of these theories has been used to describe the tissue's evolving composition and mechanical properties during growth and remodeling. Recently, a mixture theory of an arbitrary number of growing elastic materials and a fluid was derived, from which a general cartilage growth mixture model was proposed $[30,31]$. That growth mixture theory extended a series of theoretical studies of the growth of elastic biological materials [32-34]. In those theories, the deformation gradient due to growth was decomposed into two parts: a growth tensor that describes the amount and orientation of mass deposition, and an elastic accommodation tensor that ensures continuity of the tissue. Two constitutive equations must be specified for each tissue; one for the time rate of change of the growth tensor and one for the stress. Specifically, the introduction of the growth tensor required a growth law that describes how tissue deposition is regulated by mechanical stimuli such as stress, strain, strain energy, interstitial fluid velocity, etc. Furthermore, the stress depended on only the elastic component of the total deformation gradient tensor. Related theories of volumetric growth have also been recently proposed for thermoelastic materials [35] and for mixtures [36].

The general objectives of the study presented in this paper were: (1) to present a cartilage growth mixture model and (2) to use this model to solve an equilibrium boundary-value problem to illustrate the main features of the model. The specific boundaryvalue problem was chosen to illustrate how a mechanical theory of cartilage growth may be used in practice so it is constructed to mirror current experimental protocol. Currently available experimental data are not sufficient to determine all of the model's parameters and constitutive equations, so the example presented here requires a number of simplifying assumptions that are not intrinsic to the model. Consequently, the results presented are not intended as either a validation study or as a comprehensive description of the biomechanics of growth of developing cartilage. However, this paper does outline a quantitative method that may be used to interpret existing experimental data and suggests the types of experimental studies that are needed to further refine the model.

\section{Methods}

Cartilage Growth Mixture Model. The general theory of growth for a mixture of an arbitrary number of growing thermoelastic materials and a single fluid derived in [30,31] is summarized in the Appendix. The structure of that theory was motivated by how it will most readily be applied in practice. Specifically, tissue specimens are typically harvested at different stages of an in vivo or an in vitro growth process, and the tissue's compositional, geometric, and material properties can be experimentally characterized. In order to characterize how these properties evolve, all of the experimental data must be defined relative to a single reference configuration. Therefore, we introduce a fixed reference configuration that can be identified with one experimental configuration of the material, and can be used as a reference configuration for the growth boundary-value problem. In this model, the fixed reference configuration must represent some state of the growing tissue in which a proteoglycan-collagen matrix has formed. ${ }^{1}$

The cartilage growth mixture model presented here is obtained from the general theory $[30,31]$ by introducing several simplifying assumptions. First, the cartilage is modeled as a mixture of three constituents. An inviscid fluid constituent represents the water (including dissolved molecules) and two growing elastic constituents represent the proteoglycan and collagen (including noncollagenous proteins) constituents of a saturated solid matrix.

Second, two types of internal constraints that are relevant to cartilage are used. The internal constraint of solid-fluid intrinsic incompressibility, first derived in [38], is assumed. This constraint is often used in cartilage mechanics [28,39] has been demonstrated experimentally for physiological load levels [40]. The second constraint follows from the assumption that all of the individual proteoglycan and collagen molecules are bound to the extracellular solid matrix, so that their displacements and, consequently, their total deformations are equal. This is a limitation of the model, as $20-40 \%$ of the proteoglycans are soluble and mobile in the tissue matrix [41,42]; however, this feature can be added to model as the growth mixture theory presented in the appendix is sufficiently general to allow for the specification of additional mobile constituents.

Third, it is assumed that the proteoglycan constituent does not make a direct contribution to the solid matrix shear modulus; consequently, the proteoglycan stress tensor is assumed to be spherical. In particular, other authors have hypothesized that an increase in swelling pressure due to a change in proteoglycan mass produces a greater tensile stress in the collagen fibers, allowing the cross-linked collagen network to better resist shear loading (see, for example, [43]). As discussed below, the model presented in this paper does allow the proteoglycan to indirectly contribute to the solid matrix shear modulus in this manner.

Also, the model allows intrinsic remodeling of the collagen constituent. This is accomplished by including a scalar remodeling variable that may quantify an evolving microstructural property, such as collagen crosslink density, that affects the material properties that appear in the constitutive equation for the collagen stress.

To simplify the presentation, only equilibrium configurations before and after a continuous growth process are considered and

${ }^{1}$ The theory does not require the reference configuration to be physically attainable, but only that a local mapping of material points is known. The theory is general enough to allow for the stress constitutive equations for the growing materials to be defined by considering an evolving stress-free configuration by using constitutive equations for residually stressed elastic materials [37] and the growth theory for an elastic material with a residual stress field [34]. 
only the mechanical aspects of growth are discussed. The superscripts $\mathrm{p}, \mathrm{c}$, and $\mathrm{w}$ refer to the proteoglycan, collagen, and water constituents, respectively.

Kinematics. Let $\kappa_{\mathrm{R}}(\mathcal{B})$ be a fixed reference configuration and $\kappa(\mathcal{B})$ a time-dependent loaded configuration of a growing mixture $\mathcal{B}$ during a continuous growth process. The deformation gradients $\mathbf{F}^{\alpha}$ describe the overall deformations for the growing solid constituents relative to a fixed reference configuration and are decomposed as (see A.1)

$$
\mathbf{F}^{\mathrm{p}}=\mathbf{M}_{\mathrm{e}}^{\mathrm{p}} \mathbf{M}_{\mathrm{g}}^{\mathrm{p}}, \quad \mathbf{F}^{\mathrm{c}}=\mathbf{M}_{\mathrm{e}}^{\mathrm{c}} \mathbf{M}_{\mathrm{g}}^{\mathrm{c}} .
$$

The tensor $\mathbf{M}_{\mathrm{e}}^{\alpha} \mathbf{M}_{\mathrm{g}}^{\alpha}$ describes the total deformation due to growth relative to $\kappa_{\mathrm{R}}(\mathcal{B})$, where the amount and orientation of mass deposition are described by $\mathbf{M}_{\mathrm{g}}^{\alpha}$. The tensor $\mathbf{M}_{\mathrm{e}}^{\alpha}$ describes an elastic tensor that ensures continuity of the growing body, and may include a contribution arising from a superposed elastic deformation. In this theory, the tensors $\mathbf{M}_{\mathrm{e}}^{\alpha}$ and $\mathbf{M}_{\mathrm{g}}^{\alpha}$ are introduced relative to a fixed reference configuration and, consequently, lack a clear physical interpretation. However, when the current configuration is chosen as the reference configuration for a small increment of growth, these tensors have clear physical meanings as shown in [34].

As discussed above, two internal constraints are used. The first constraint is solid-fluid intrinsic incompressibility [38]:

$$
\left(\rho^{\mathrm{p}} / \rho^{\mathrm{pT}}\right)+\left(\rho^{\mathrm{c}} / \rho^{\mathrm{cT}}\right)+\left(\rho^{\mathrm{w}} / \rho^{\mathrm{wT}}\right)=1,
$$

where $\rho^{\alpha}$ is the apparent density (per tissue volume) and $\rho^{\alpha T}$ is the true density (per constituent volume). The second constraint is derived by assuming that all of the proteoglycan and collagen molecules are bound to the extracellular solid matrix, so that their total deformations are equal: ${ }^{2}$

$$
\mathbf{F}^{\mathrm{p}}=\mathbf{F}^{\mathrm{c}} \Rightarrow \mathbf{M}_{\mathrm{e}}^{\mathrm{p}} \mathbf{M}_{\mathrm{g}}^{\mathrm{p}}=\mathbf{M}_{\mathrm{e}}^{\mathrm{c}} \mathbf{M}_{\mathrm{g}}^{\mathrm{c}} .
$$

Balance Equations. For the proteoglycan and collagen constituents, the reduced continuity equations are (see $A 6)^{3}$

$$
\rho^{\mathrm{p}} \operatorname{det} \mathbf{M}_{\mathrm{e}}^{\mathrm{p}}=\rho_{\mathrm{R}}^{\mathrm{p}}, \quad \rho^{\mathrm{c}} \operatorname{det} \mathbf{M}_{\mathrm{e}}^{\mathrm{c}}=\rho_{\mathrm{R}}^{\mathrm{c}} .
$$

Here, $\rho_{\mathrm{R}}^{\alpha}$ is the apparent density in the reference configuration. Also, the growth continuity equations are (see $A 7)$

$$
\operatorname{det} \mathbf{M}_{\mathrm{g}}^{\mathrm{p}}=\exp \left\lfloor\int_{\tau=\mathrm{t}_{0}}^{\mathrm{t}} \mathrm{c}^{\mathrm{p}} \mathrm{d} \tau\right\rfloor, \quad \operatorname{det} \mathbf{M}_{\mathrm{g}}^{\mathrm{c}}=\exp \left\lfloor\int_{\tau=\mathrm{t}_{0}}^{\mathrm{t}} \mathrm{c}^{\mathrm{c}} \mathrm{d} \tau\right\rfloor,
$$

where $\mathrm{c}^{\alpha}$ is the mass growth function (the rate of mass deposition per unit current mass). At equilibrium, it is assumed that the diffusive forces vanish. Neglecting body forces, the equilibrium equations become (see $A 3$ )

$$
\operatorname{div} \mathbf{T}^{\mathrm{p}}=\mathbf{0}, \quad \operatorname{div} \mathbf{T}^{\mathrm{c}}=\mathbf{0}, \quad \operatorname{div} \mathbf{T}^{\mathrm{w}}=\mathbf{0} .
$$

The additional balance equations that appear in the general theory are listed in the Appendix.

Stress Constitutive Equations. Constitutive equations are required for the determinate parts of the proteoglycan and collagen stresses that appear in (6). In general, the constituent stresses and the tissue's material properties will change during growth due to changes in both the composition of the tissue and the structure of the individual constituents. As in any continuum theory of growth, these constitutive equations can be defined relative to an evolving virtual configuration using the methods outlined in [34] and the constitutive equations for residually stressed elastic materials developed in [37]. Specific forms of the stress constitutive equations are postulated below for the boundary-value problem that is stud-

\footnotetext{
${ }^{2}$ The constraints $(2-3)$ lead to the indeterminate stress and diffusive forces responses $(A 12-A 13)$.

${ }^{3} \mathrm{Also}$, a continuity equation for the fluid constituent is required (see $A 2$ ).
}

ied. It should be emphasized that the specific constitutive equations used here are not intrinsic to the cartilage growth model, and that different equations will generally be required for solving different problems. For example, the stress constitutive equations can vary with anatomical location and also with the stage of development, ageing, or degeneration.

In the mixture theory formulation, the solid matrix stress $\mathbf{T}^{\mathrm{s}}$ is the sum of the proteoglycan and collagen stresses:

$$
\mathbf{T}^{\mathrm{s}}=\mathbf{T}^{\mathrm{p}}+\mathbf{T}^{\mathrm{c}}
$$

Consequently, the proteoglycan and collagen stress response functions can be used to construct the stress constitutive equation for the solid matrix. Due to the internal constraints, the indeterminate parts of the proteoglycan and collagen stresses are (see $A 12$ and A13)

$$
\widetilde{\mathbf{T}}^{\mathrm{p}}=\widetilde{\boldsymbol{\lambda}}+\mathrm{p} \frac{\rho^{\mathrm{p}}}{\rho^{\mathrm{pT}}} \mathbf{1}, \quad \widetilde{\mathbf{T}}^{\mathrm{c}}=-\widetilde{\boldsymbol{\lambda}}+\mathrm{p} \frac{\rho^{\mathrm{c}}}{\rho^{\mathrm{cT}}} \mathbf{1},
$$

respectively. ${ }^{4}$ At equilibrium, it is assumed that the indeterminate second-order tensor $\tilde{\boldsymbol{\lambda}}$ vanishes. However, the tensors $\tilde{\boldsymbol{\lambda}}$ that appear in (8) can be seen to have an important implication. Note that, when adding the proteoglycan and collagen stresses to form the solid matrix stress, the tensors $\tilde{\boldsymbol{\lambda}}$ and $-\tilde{\boldsymbol{\lambda}}$ cancel. Consequently, the governing equations can be satisfied by ensuring that the equilibrium equations for the solid matrix

$$
\operatorname{div} \mathbf{T}^{\mathrm{s}}=\mathbf{0}
$$

are satisfied while the arbitrariness of the tensor $\tilde{\boldsymbol{\lambda}}$ ensures that the equilibrium equations $(6)_{1,2}$ can be satisfied individually. ${ }^{5}$

The Growth Tensor and the Growth Law. To obtain a complete theory, growth laws that describe the time-rate of change of the growth tensors $\mathbf{M}_{\mathrm{g}}^{\alpha}$ for both the proteoglycan and collagen constituents must be specified (see A10). The growth law provides a description of the rate at which material is deposited (or resorbed) at a point, the orientation in which material deposition occurs, and the way in which mechanical factors influence mass deposition. For soft biological tissues, Taber and colleagues (e.g., see [21]) and Van Dyke and Hoger [33] have implemented growth laws that depend on stress, with the possibility of including a homeostatic stress. However, it is possible that the growth law should depend on other mechanical stimuli, such as the strain, strain energy, the rate of strain, and interstitial fluid velocity. Constructing complete growth laws of the form (A10) for cartilage would require substantially more information than that which is currently available. In this paper, experimental data is used to calculate the ratio of the determinants of the growth tensors at different developmental stages.

Qualitative Example. Before solving a specific boundaryvalue problem in the next section, here we present a simple qualitative example in order to illustrate how the cartilage growth mixture model can describe the remodeling of the composition and the mechanical properties of cartilage due to mass deposition of the solid matrix constituents. This example is physically unrealistic, but is intended to illustrate the main features of the model.

Consider a special case of growth of an unloaded, homogeneous spherical element of cartilage for which only proteoglycan molecules are deposited (Fig. 2). When uniform proteoglycan mass deposition occurs, the stress supported by the proteoglycan constituent becomes more compressive due to the increase in fixed charge density. In order to achieve a new, unloaded equilibrium state for which there is a balance of stresses, the stress supported by the collagen network must become more tensile and, consequently, a local tissue expansion will occur. This physical description is outlined schematically in Fig. 2A. To describe this growth process mathematically, the cartilage growth mixture

\footnotetext{
${ }^{4}$ Also, the diffusive forces have indeterminate terms as discussed in the Appendix.

${ }^{5}$ This observation extends to non-equilibrium conditions and was also suggested in the constrained growth mixture theory developed in [36].
} 


\section{A: Physical description of growth}
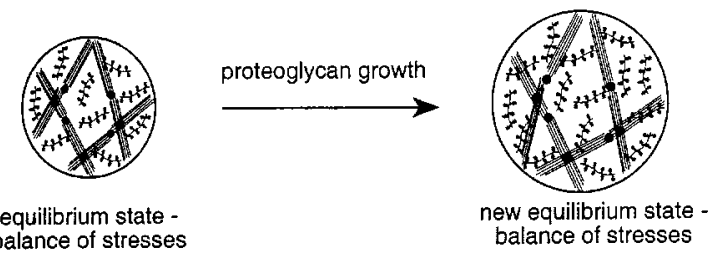

equilibrium state balance of stresses

\section{B: Mathematical description of growth}

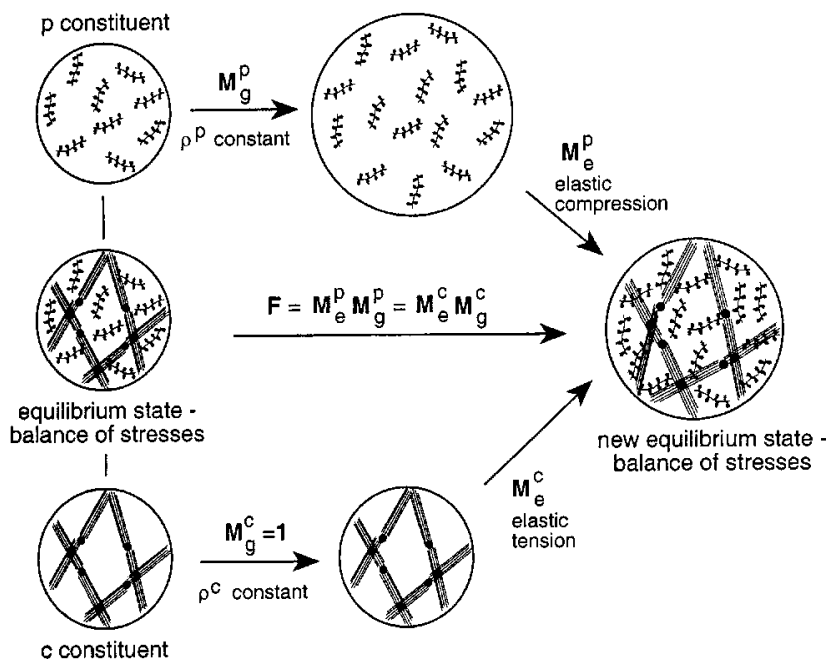

Fig. 2 Physical and mathematical descriptions of cartilage growth for the special case when only proteoglycan molecules are deposited. See accompanying text for a full description.

model introduces intermediate configurations for the individual constituents. These intermediate configurations describe the amount and orientation of mass deposition for the individual constituents in which the constituent densities remain unchanged. In this special example, proteoglycan growth leads to the intermediate configuration defined by the growth tensor $\mathbf{M}_{\mathrm{g}}^{\mathrm{p}}$ (Fig. 2B). The tensor $\mathbf{M}_{\mathrm{g}}^{\mathrm{c}}$ is equal to the identity tensor since no collagen growth occurs. These intermediate configurations clearly do not represent a continuous solid matrix; consequently, in the mathematical description of growth elastic tensors $\mathbf{M}_{\mathrm{e}}^{\mathrm{p}}$ and $\mathbf{M}_{\mathrm{e}}^{\mathrm{c}}$ are introduced that must satisfy the constraint (3) and the equilibrium equations for the solid matrix (9). In order to solve this simple boundary-value problem, stress constitutive equations for the proteoglycan and collagen constituents must be specified. Once $\mathbf{M}_{\mathrm{e}}^{\mathrm{p}}$ and $\mathbf{M}_{\mathrm{e}}^{\mathrm{c}}$ are determined, the proteoglycan and collagen densities can be determined from the continuity equations (4) and the apparent fluid density can be determined from the intrinsic incompressibility constraint (2). When nonlinear constitutive equations are used, the solid matrix stress response function relative to the growth configuration will, in general, change. In this simple example, the tensile collagen strain produced by the growth process may lead to higher tensile and shear moduli as measured by conducting experiments on the grown configuration. Thus, this mathematical description of growth reveals how the cartilage growth model can describe the evolution of the tissue's compositional and mechanical properties during growth.

The Equilibrium Boundary-Value Problem. Here, an equilibrium boundary-value problem that describes cartilage growth is formulated. The specific boundary-value problem that is solved is chosen due to its simplicity in order to illustrate how the governing equations can be used together to describe growth-related experiments on cartilage explants. Typically, the biomechanical and biochemical changes during an in vivo growth process are quan- tified by harvesting tissue samples of the same size and shape at different developmental or degeneration stages and conducting experiments on the tissue. Since many of the model parameters and constitutive equations that describe an in vivo growth process are presently unknown, the boundary-value problem that is presented here only indirectly reflects the in vivo growth process. Specifically, it is aimed at quantifying the evolution of the biochemical and biomechanical properties of unloaded tissue explants that are harvested at different developmental stages of a continuous in vivo growth process.

Formulation. Since the purpose of this example is to illustrate how the theory may be applied in practice and since only limited experimental data for the mechanical properties were available, simple constitutive equations for the proteoglycan and collagen constituents were used for which the material properties are assumed to be uniform and isotropic. In addition, it is assumed that the growth tensors are uniform and isotropic. The boundary-value problem developed here describes the initial and grown configurations of the cartilage explant in equilibrium in the absence of external loads. ${ }^{6}$ If the tissue explants are small enough, then the solid matrix stress and the fluid pore pressure for an unloaded configuration will be close to zero; consequently, this study describes how the stress-free configuration of the tissue changes during developmental growth.

Since experiments have not been conducted that follow the same control volume of growing cartilage, it is not possible to completely characterize the growth tensors of the individual constituents. However, it is possible to estimate the magnitude of the elastic accommodation tensors and the growth ratio as follows. The elastic accommodation tensors are uniform and isotropic; they are assumed to be of the form

$$
\mathbf{M}_{\mathrm{e}}^{\mathrm{p}}=\lambda_{\mathrm{e}}^{\mathrm{p}} \mathbf{1}, \quad \mathbf{M}_{\mathrm{e}}^{\mathrm{c}}=\lambda_{\mathrm{e}}^{\mathrm{c}} \mathbf{1},
$$

where $\lambda_{\mathrm{e}}^{\alpha}$ are called the elastic stretches. Using experimentally measured densities $\rho^{\alpha}$ and $\rho_{\mathrm{R}}^{\alpha}$, the determinants of the elastic accommodation tensors and, consequently, the elastic stretches are calculated from (4) as

$$
\operatorname{det} \mathbf{M}_{\mathrm{e}}^{\mathrm{p}}=\left(\lambda_{\mathrm{e}}^{\mathrm{p}}\right)^{3}=\rho_{\mathrm{R}}^{\mathrm{p}} / \rho^{\mathrm{p}}, \quad \operatorname{det} \mathbf{M}_{\mathrm{e}}^{\mathrm{c}}=\left(\lambda_{\mathrm{e}}^{\mathrm{c}}\right)^{3}=\rho_{\mathrm{R}}^{\mathrm{c}} / \rho^{\mathrm{c}} .
$$

Then, the growth ratio $G^{\mathrm{p} / \mathrm{c}}$ is calculated from (3) and (11):

$$
\mathrm{G}^{\mathrm{p} / \mathrm{c}}=\operatorname{det} \mathbf{M}_{\mathrm{g}}^{\mathrm{p}} / \operatorname{det} \mathbf{M}_{\mathrm{g}}^{\mathrm{c}}=\operatorname{det} \mathbf{M}_{\mathrm{e}}^{\mathrm{c}} / \operatorname{det} \mathbf{M}_{\mathrm{e}}^{\mathrm{p}} .
$$

The fluid stress is assumed to be zero at equilibrium. Given our assumptions that the material properties are uniform, the growth and elastic tensors are uniform and isotropic, and that the tissue explant has a no-load boundary condition, the equilibrium equation for the solid constituent (9) requires that the total solid matrix stress vanishes everywhere:

$$
\mathbf{T}^{\mathrm{s}}=\mathbf{T}^{\mathrm{p}}+\mathbf{T}^{\mathrm{c}}=\mathbf{0} .
$$

To determine the proteoglycan and collagen stresses that appear in (13), specific constitutive equations must be used (the indeterminate stress terms are assumed to vanish at equilibrium). Since the in vivo growth process potentially corresponds to finite growth and elastic strains, nonlinear constitutive equations are used. Typically, experiments that apply a known deformation to the tissue explant and measure the stress response may be used to determine the mechanical properties for finite deformations of the solid matrix. Currently, a stress constitutive equation for the solid matrix of articular cartilage validated for general finite deformations is lacking. Therefore, only the linear elastic response of the solid matrix relative to an equilibrium configuration is used to determine the material constants in the collagen stress constitutive equations that are assumed in this paper to hold for the continuous growth process. In particular, two linear elastic material constants for the solid matrix are used: the aggregate modulus determined

${ }^{6}$ Due to these assumptions, the growth, elastic, and stress tensors are all spherical 
from a confined compression experiment and the Poisson's ratio determined from an unconfined compression experiment.

For the proteoglycan constituent, it is assumed that the stress response function depends on the changes in the constituent densities during the growth process. As discussed above, it is assumed that the proteoglycan constituent does not have a direct contribution to the solid matrix shear modulus; consequently, the proteoglycan stress tensor is assumed to be spherical. Generally, the proteoglycan stress constitutive equation relative to any configuration $\kappa(\mathcal{B})$ is assumed to be of the form

$$
\mathbf{T}^{\mathrm{p}}=\hat{\mathbf{T}}_{\kappa}^{\mathrm{p}}=\hat{\mathrm{T}}_{\kappa}^{\mathrm{p}}\left(\rho^{\mathrm{p}}, \rho^{\mathrm{c}}, \rho^{\mathrm{w}}\right) \mathbf{1} .
$$

An explicit formula of the form (14) that holds during the continuous growth process is not proposed; instead, the proteoglycan stress and aggregate modulus for a superposed confined compression experiment for each tissue explant are derived using the model of [4]. For each explant, the constituent densities and solid matrix aggregate modulus $\bar{H}_{\mathrm{A}}^{\mathrm{s}}$ are used to calculate the proteoglycan stress component $\mathrm{T}_{\kappa}^{\mathrm{p}}$ and aggregate modulus $\bar{H}_{\mathrm{A}}^{\mathrm{p}}{ }^{7}$ Thus, at each stage of a continuous growth process for which the constituent densities and $\bar{H}_{\mathrm{A}}^{\mathrm{s}}$ can be experimentally determined, using the model of [4] we can construct a linear elastic constitutive equation relative to the grown configuration of the form:

$$
\mathbf{T}^{\mathrm{p}}=\bar{T}_{\kappa}^{\mathrm{p}}\left(\rho^{\mathrm{p}}, \rho^{\mathrm{c}}, \rho^{\mathrm{w}}\right) \mathbf{1}=\overline{\mathrm{H}}_{\mathrm{A}}^{\mathrm{p}}(\operatorname{tre}) \mathbf{1}+\mathrm{T}_{\kappa}^{\mathrm{p}} \mathbf{1}
$$

where $\mathbf{e}$ is the solid matrix infinitesimal elastic strain tensor relative to the grown configuration $\kappa(\mathcal{B}),{ }^{8} T_{\kappa}^{\mathrm{p}}$ is the proteoglycan stress in the configuration $\kappa(\mathcal{B})$, and $\bar{H}_{\mathrm{A}}^{\mathrm{p}}$ is the proteoglycan aggregate modulus.

As in previous descriptions of volumetric growth of elastic materials, we take the collagen stress constitutive equation to depend on the elastic part of the deformation gradient, $\mathbf{M}_{\mathrm{e}}^{\mathrm{c}}[20,21,32,34]$. Here, however, we allow the material properties of the collagen constituent to change during the growth process through the evolution of a remodeling variable $\gamma^{\mathrm{c}}$. In this study, this remodeling variable represents the change in crosslink density. Because the goal of this study is to illustrate how the growth model may be used in practice, the constitutive equations used in this study were chosen for their simplicity. To accurately reflect the growth of cartilage, better constitutive equations will have to be developed. Furthermore, we considered two nonlinear stress constitutive equations to explore the sensitivity of the model to the choice of constitutive equation. Because the experimental data that was available was limited, the number of material constants was kept to a minimum.

CASE A: The first collagen stress constitutive equation is a Saint-Venant Kirchhoff material defined in terms of the Biot strain tensor with an initial spherical stress. This equation was chosen because it is the most simple equation that can be used for large deformations of an elastic material; in particular, the stress is firstorder in the right stretch tensor. This equation is obtained by neglecting the second-order terms in the corresponding equation derived in [44]:

$$
\mathbf{T}^{\mathrm{c}}=\hat{\mathbf{T}}_{\kappa_{\mathrm{R}}}^{\mathrm{c}}=\mathbf{R}_{\mathrm{e}}^{\mathrm{c}}\left\{\lambda^{\mathrm{c}}\left(\operatorname{tr} \mathbf{E}_{\mathrm{B}}^{\mathrm{c}}\right) \mathbf{1}+2 \mu^{\mathrm{c}} \mathbf{E}_{\mathrm{B}}^{\mathrm{c}}+\Gamma^{\mathrm{c}} \mathbf{1}\right\} \mathbf{R}_{\mathrm{e}}^{\mathrm{c}^{\mathrm{T}}},
$$

where $\left(\lambda^{\mathrm{c}}, \mu^{\mathrm{c}}, \Gamma^{\mathrm{c}}\right)$ are collagen material constants, $\mathbf{R}_{\mathrm{e}}^{\mathrm{c}}$ is the rotation tensor associated with $\mathbf{M}_{\mathrm{e}}^{\mathrm{c}}$, and $\mathbf{E}_{\mathrm{B}}^{\mathrm{c}}$ is the Biot strain tensor

$$
\mathbf{E}_{\mathrm{B}}^{\mathrm{c}}=\mathbf{U}_{\mathrm{e}}^{\mathrm{c}}-\mathbf{1},
$$

where $\mathbf{U}_{\mathrm{e}}^{\mathrm{c}}$ is the right stretch tensor associated with $\mathbf{M}_{\mathrm{e}}^{\mathrm{c}}$. In the reference configuration where $\mathbf{U}_{\mathrm{e}}^{\mathrm{c}}=\mathbf{1}, \mathbf{E}_{\mathrm{B}}^{\mathrm{c}}=\mathbf{0}$, and $\mathbf{R}_{\mathrm{e}}^{\mathrm{c}}=\mathbf{1}$, the value $\hat{\mathbf{T}}_{\kappa_{\mathrm{R}}}^{\mathrm{c}}=\Gamma^{\mathrm{c}} \mathbf{1}$ is the initial collagen stress. The collagen material

\footnotetext{
${ }^{7}$ Quantities with a superposed bar indicate either material constants or stress response functions measured relative to the present configuration of the growing tissue.

${ }^{8}$ For a superimposed infinitesimal elastic strain, the proteoglycan and collagen elastic strains are equal to $\mathbf{e}$.
}

constants may depend explicitly on collagen crosslink density $\gamma^{\mathrm{c}}$, so that both the collagen material properties and the collagen stress will change as the collagen remodels. Note that if remodeling occurs while the collagen elastic strain is held fixed, then (16) suggests that the parameter $\Gamma^{\mathrm{c}}$ should be allowed to change so that the collagen stress may change. Since we are also interested in studying the linear elastic response of the solid matrix relative to the grown configuration, a "small on large" approach was used to obtain the required constitutive equation following a finite growth. For a material that is either residually or initially stressed, the general approach is outlined in [22]. The required constitutive equation for a solid matrix infinitesimal elastic strain e superimposed on an isotropic growth $\mathbf{M}_{\mathrm{e}}^{\mathrm{c}}=\lambda_{\mathrm{e}}^{\mathrm{c}} \mathbf{1}$ can be expressed as

$$
\mathbf{T}^{\mathrm{c}}=\overline{\mathbf{T}}_{\kappa}^{\mathrm{c}}=\bar{\lambda}^{\mathrm{c}}(\operatorname{tr} \mathbf{e}) \mathbf{1}+2 \bar{\mu}^{\mathrm{c}} \mathbf{e}+\bar{\Gamma}^{\mathrm{c}} \mathbf{1}
$$

where the material constants $\left(\bar{\lambda}^{\mathrm{c}}, \bar{\mu}^{\mathrm{c}}, \bar{\Gamma}^{\mathrm{c}}\right)$ defined relative to the grown configuration are functions of the initial material constants $\left(\lambda^{\mathrm{c}}, \mu^{\mathrm{c}}, \Gamma^{\mathrm{c}}\right)$ and the elastic stretch due to growth, $\lambda_{\mathrm{e}}^{\mathrm{c}}$ :

$$
\begin{gathered}
\bar{\lambda}^{\mathrm{c}}=\left(\lambda_{\mathrm{e}}^{\mathrm{c}}\right) \lambda^{\mathrm{c}}, \quad \bar{\mu}^{\mathrm{c}}=\left(\lambda_{\mathrm{e}}^{\mathrm{c}}\right) \mu^{\mathrm{c}}, \\
\bar{\Gamma}^{\mathrm{c}}=3\left(\lambda_{\mathrm{e}}^{\mathrm{c}}-1\right) \lambda^{\mathrm{c}}+2\left(\lambda_{\mathrm{e}}^{\mathrm{c}}-1\right) \mu^{\mathrm{c}}+\Gamma^{\mathrm{c}} .
\end{gathered}
$$

CASE B: The second constitutive equation was chosen due to its familiarity in the mechanics literature; it is the classical SaintVenant Kirchhoff material that is generalized to include an initial spherical stress [45]:

$$
\mathbf{T}^{\mathrm{c}}=\hat{\mathbf{T}}_{\kappa_{\mathrm{R}}}^{\mathrm{c}}=\frac{\rho^{\mathrm{c}}}{\rho_{\mathrm{R}}^{\mathrm{c}}} \mathbf{M}_{\mathrm{e}}^{\mathrm{c}}\left\{\lambda^{\mathrm{c}}\left(\operatorname{tr} \mathbf{E}^{\mathrm{c}}\right) \mathbf{1}+2 \mu^{\mathrm{c}} \mathbf{E}^{\mathrm{c}}+\Gamma^{\mathrm{c}} \mathbf{1}\right\} \mathbf{M}_{\mathrm{e}}^{\mathrm{c}^{\mathrm{T}}},
$$

where $\left(\lambda^{\mathrm{c}}, \mu^{\mathrm{c}}, \Gamma^{\mathrm{c}}\right)$ are collagen material constants and $\mathbf{E}^{\mathrm{c}}$ is the Lagrangean strain tensor derived from

$$
\mathbf{E}^{\mathrm{c}}=\frac{1}{2}\left(\mathbf{M}_{\mathrm{e}}^{\mathrm{c}^{\mathrm{T}}} \mathbf{M}_{\mathrm{e}}^{\mathrm{c}}-\mathbf{1}\right) .
$$

Although the constitutive equations (16) and (20) appear to be similar, we will see that they do provide quite different results. In a reference configuration where $\mathbf{M}_{\mathrm{e}}^{\mathrm{c}}=\mathbf{1}, \mathbf{E}^{\mathrm{c}}=\mathbf{0}$, and $\rho^{\mathrm{c}}=\rho_{\mathrm{R}}^{\mathrm{c}}$, the value $\hat{\mathbf{T}}_{\kappa_{\mathrm{R}}}^{\mathrm{c}}=\Gamma^{\mathrm{c}} \mathbf{1}$ is the initial collagen stress. As before, the collagen material constants may depend explicitly on collagen crosslink density $\gamma^{\mathrm{c}}$. Also, the required constitutive equation for an infinitesimal elastic strain of the solid matrix e superimposed on an isotropic growth $\mathbf{M}_{\mathrm{e}}^{\mathrm{c}}=\lambda_{\mathrm{e}}^{\mathrm{c}} \mathbf{1}$ has the same form as (18); however, the material constants $\left(\bar{\lambda}^{\mathrm{c}}, \bar{\mu}^{\mathrm{c}}, \bar{\Gamma}^{\mathrm{c}}\right)$ are defined as different functions of the initial material constants $\left(\lambda^{\mathrm{c}}, \mu^{\mathrm{c}}, \Gamma^{\mathrm{c}}\right)$ and the elastic stretch due to growth, $\lambda_{\mathrm{e}}^{\mathrm{c}}$ :

$$
\begin{aligned}
& \bar{\lambda}^{\mathrm{c}}=\frac{1}{\lambda_{\mathrm{e}}^{\mathrm{c}}}\left[\left(1.5-0.5 \lambda_{\mathrm{e}}^{\mathrm{c}^{2}}\right) \lambda^{\mathrm{c}}-\left(\lambda_{\mathrm{e}}^{\mathrm{c}^{2}}-1\right) \mu^{\mathrm{c}}-\Gamma^{\mathrm{c}}\right], \\
& \bar{\mu}^{\mathrm{c}}=\frac{1}{\lambda_{\mathrm{e}}^{\mathrm{c}}}\left[1.5\left(\lambda_{\mathrm{e}}^{\mathrm{c}^{2}}-1\right) \lambda^{\mathrm{c}}+\left(2 \lambda_{\mathrm{e}}^{\mathrm{c}^{2}}-1\right) \mu^{\mathrm{c}}+\Gamma^{\mathrm{c}}\right], \\
& \bar{\Gamma}^{\mathrm{c}}=\frac{1}{\lambda_{\mathrm{e}}^{\mathrm{c}}}\left[1.5\left(\lambda_{\mathrm{e}}^{\mathrm{c}^{2}}-1\right) \lambda^{\mathrm{c}}+\left(\lambda_{\mathrm{e}}^{\mathrm{c}^{2}}-1\right) \mu^{\mathrm{c}}+\Gamma^{\mathrm{c}}\right] .
\end{aligned}
$$

Comparison of (22) with (19) reveals an important difference in the two constitutive equations that were chosen. In particular, the dependence of the material constants $\left(\bar{\lambda}^{\mathrm{c}}, \bar{\mu}^{\mathrm{c}}, \bar{\Gamma}^{\mathrm{c}}\right)$ defined relative to the grown configuration on the collagen elastic stretch is linear for CASE A and nonlinear for CASE B.

Use of Experimental Data and Solution of the BoundaryValue Problem. Constituent densities, solid matrix aggregate modulus, and crosslink density from third trimester fetal (F,n $=6), 1-3$ week old newborn $(\mathrm{N}, \mathrm{n}=8)$, and $1.5-2$ year old adult $(\mathrm{A}, \mathrm{n}=7)$ bovine articular cartilage specimens $(1 \mathrm{~mm}$ thick with intact articular surfaces) harvested from the patellofemoral groove 
Table 1 Mean \pm S.D. values for the proteoglycan density $\rho^{p}$, collagen density $\rho^{\mathrm{c}}$, water density $\rho^{\mathrm{w}}$, normalized crosslink density $\gamma^{c}$, and solid matrix aggregate modulus $\overline{\mathrm{H}}_{\mathrm{A}}^{\mathrm{s}}$ and for the fetal $(F, n=6)$, newborn, $(N, n=8)$, and adult $(A, n=7)$ specimens. Crosslink density values were normalized by dividing each value by the average of the $F$ group. This data was available from $[15,46]$.

\begin{tabular}{cccccc}
\hline \hline Group & $\begin{array}{c}\rho^{\mathrm{p}} \\
\left(\mathrm{mg} / \mathrm{cm}^{3}\right)\end{array}$ & $\begin{array}{c}\rho^{\mathrm{c}} \\
\left(\mathrm{mg} / \mathrm{cm}^{3}\right)\end{array}$ & $\begin{array}{c}\rho^{\mathrm{w}} \\
\left(\mathrm{mg} / \mathrm{cm}^{3}\right)\end{array}$ & $\gamma^{\mathrm{c}}$ & $\begin{array}{c}\overline{\mathrm{H}}_{\mathrm{A}}^{\mathrm{s}} \\
(\mathrm{MPa})\end{array}$ \\
\hline $\mathrm{F}$ & 22.2 & 87.6 & 963 & 1.0 & 0.09 \\
& $(2.1)$ & $(19.6)$ & $(18.2)$ & $(0.5)$ & $(0.03)$ \\
$\mathrm{N}$ & 26.0 & 141 & 942 & 2.1 & 0.28 \\
& $(4.3)$ & $(18.4)$ & $(9.4)$ & $(0.6)$ & $(0.05)$ \\
$\mathrm{A}$ & 23.3 & 183 & 902 & 2.3 & 0.31 \\
& $(3.6)$ & $(28.8)$ & $(22.8)$ & $(1.1)$ & $(0.19)$ \\
\hline \hline
\end{tabular}

were available [15,46] (Table 1). Collagen crosslink density data was converted into units of moles pyridinoline per mole collagen, and then normalized by the average value for fetal specimens. The reference configuration for all specimens was defined by the "average" fetal specimen. In particular, the reference densities $\rho_{\mathrm{R}}^{\alpha}$ are equal to the average density values from the fetal specimens. In the reference configuration, the elastic and growth tensors are equal to the identity tensors. For each explant, the elastic stretches and the growth ratio were calculated using the density data and (11-12).

Then, the constituent densities $\rho^{\alpha}$ and solid matrix aggregate modulus $\overline{\mathrm{H}}_{\mathrm{A}}^{\mathrm{s}}$ were used to calculate the proteoglycan stress component $\mathrm{T}_{\kappa}^{\mathrm{p}}$ and aggregate modulus $\overline{\mathrm{H}}_{\mathrm{A}}^{\mathrm{p}}$ using the model of [4] for each explant. The three collagen material constants $\left(\lambda^{\mathrm{c}}, \mu^{\mathrm{c}}, \Gamma^{\mathrm{c}}\right)$ were determined from solving three equations simultaneously. The first equation was obtained by equating the collagen stress $\mathbf{T}^{\mathrm{c}}=T_{\kappa}^{\mathrm{c}} \mathbf{1}=\bar{\Gamma}^{\mathrm{c}} \mathbf{1}$ determined from (18) and (19) ${ }_{3}$ (CASE A) or $(22)_{3}$ (CASE B) and the collagen stress determined from (13) using $\mathrm{T}_{\kappa}^{\mathrm{p}}$. The second equation corresponded to the solid matrix aggregate modulus $\overline{\mathrm{H}}_{\mathrm{A}}^{\mathrm{s}}$; in this model, $\overline{\mathrm{H}}_{\mathrm{A}}^{\mathrm{s}}$ is equal to the sum of the proteoglycan and collagen aggregate moduli:

$$
\overline{\mathrm{H}}_{\mathrm{A}}^{\mathrm{s}}=\overline{\mathrm{H}}_{\mathrm{A}}^{\mathrm{p}}+\overline{\mathrm{H}}_{\mathrm{A}}^{\mathrm{c}},
$$

where the relationship $\overline{\mathrm{H}}_{\mathrm{A}}^{\mathrm{c}}=\bar{\lambda}^{\mathrm{c}}+2 \bar{\mu}^{\mathrm{c}}$ can be derived easily from (18) and was calculated using the values of $\bar{\lambda}^{\mathrm{c}}$ and $\bar{\mu}^{\mathrm{c}}$ from (19) $)_{1,2}$ (CASE A) or (22) $)_{1,2}$ (CASE B). The third equation was obtained by assuming values for the solid matrix Poisson's ratio $\bar{\nu}^{\mathrm{s}}$, which using (15) and (18) may be calculated as

$$
\bar{\nu}^{\mathrm{s}}=\frac{\bar{\lambda}^{\mathrm{c}}+\overline{\mathrm{H}}_{\mathrm{A}}^{\mathrm{p}}}{2\left(\bar{\lambda}^{\mathrm{c}}+\overline{\mathrm{H}}_{\mathrm{A}}^{\mathrm{p}}+\bar{\mu}^{\mathrm{c}}\right)}
$$

using the values of $\bar{\lambda}^{\mathrm{c}}$ and $\bar{\mu}^{\mathrm{c}}$ from (19) $)_{1,2}$ (CASE A) or (22) 1,2 (CASE B). Since the tissue Poisson's ratio was not measured in the experimental study, values of $\bar{\nu}^{\mathrm{s}}$ equal to $0.09,0.11$, and 0.26

Table 2 Mean \pm S.D. values for the stress components $T_{\kappa}^{p}$ and $T_{\kappa}^{c}$ and aggregate moduli $\bar{H}_{A}^{p}$ and $\bar{H}_{A}^{c}$ for the fetal $(F, n=6)$, newborn $(N, n=8)$, and adult $(A, n=7)$ specimens. These values were calculated using the model of [4].

\begin{tabular}{ccccc}
\hline \hline Group & $\begin{array}{c}\mathrm{T}_{K}^{\mathrm{p}} \\
(\mathrm{MPa})\end{array}$ & $\begin{array}{c}\mathrm{T}_{K}^{\mathrm{c}} \\
(\mathrm{MPa})\end{array}$ & $\begin{array}{c}\overline{\mathrm{H}}_{\mathrm{A}}^{\mathrm{p}} \\
(\mathrm{MPa})\end{array}$ & $\begin{array}{c}\overline{\mathrm{H}}_{\mathrm{A}}^{\mathrm{c}} \\
(\mathrm{MPa})\end{array}$ \\
\hline $\mathrm{F}$ & -0.055 & 0.055 & 0.09 & 0.001 \\
& $(0.010)$ & $(0.010)$ & $(0.03)$ & $(0.010)$ \\
$\mathrm{N}$ & -0.074 & 0.074 & 0.13 & 0.149 \\
& $(0.015)$ & $(0.015)$ & $(0.02)$ & $(0.067)$ \\
$\mathrm{A}$ & -0.078 & 0.078 & 0.17 & 0.139 \\
& $(0.022)$ & $(0.022)$ & $(0.06)$ & $(0.149)$ \\
\hline \hline
\end{tabular}

were assumed for the F, N, and A specimens, respectively, based on data reported from unconfined compression experiments [16].

After determining the collagen material constants $\left(\lambda^{\mathrm{c}}, \mu^{\mathrm{c}}, \Gamma^{\mathrm{c}}\right)$ in this manner, the collagen material constants $\left(\bar{\lambda}^{\mathrm{c}}, \bar{\mu}^{\mathrm{c}}, \bar{\Gamma}^{\mathrm{c}}\right)$ defined relative to the grown configuration were determined from (19) (CASE A) and (22) (CASE B). Finally, the solid matrix shear modulus $\overline{\mathrm{G}}^{\mathrm{s}}$, bulk modulus $\overline{\mathrm{k}}^{\mathrm{s}}$, and Young's modulus $\overline{\mathrm{E}}^{\mathrm{s}}$ were calculated by solving the appropriate boundary-value problems using the linear elastic solid matrix constitutive equation obtained upon adding (15) and (18):

$$
\overline{\mathrm{G}}^{\mathrm{s}}=2 \bar{\mu}^{\mathrm{c}}, \quad \bar{k}^{\mathrm{s}}=\bar{\lambda}^{\mathrm{c}}+\frac{2}{3} \bar{\mu}^{\mathrm{c}}+\overline{\mathrm{H}}_{\mathrm{A}}^{\mathrm{p}}, \quad \overline{\mathrm{E}}^{\mathrm{s}}=\frac{\bar{\mu}^{\mathrm{c}}\left(3 \bar{\lambda}^{\mathrm{c}}+3 \overline{\mathrm{H}}_{\mathrm{A}}^{\mathrm{p}}+2 \bar{\mu}^{\mathrm{c}}\right)}{\bar{\lambda}^{\mathrm{c}}+\overline{\mathrm{H}}_{\mathrm{A}}^{\mathrm{p}}+\bar{\mu}^{\mathrm{c}}} .
$$

To ensure that these material constants for the solid matrix satisfy stability constraints for linear elastic materials, we verified that the shear modulus $\overline{\mathrm{G}}^{\mathrm{s}}$ and bulk modulus $\overline{\mathrm{k}}^{\mathrm{s}}$ were positive: ${ }^{9}$

Statistical Analysis. The effect of developmental stage on the model parameters was analyzed by multi-way ANOVA and posthoc Tukey test (Systat). Data are shown as mean \pm standard error of the mean. The dependence of the collagen and solid matrix material constants on $\gamma^{\mathrm{c}}$ was analyzed by linear regression.

\section{Results}

The calculated values for the proteoglycan and collagen stress components $\mathrm{T}_{\kappa}^{\mathrm{p}}$ and $\mathrm{T}_{\kappa}^{\mathrm{c}}$ and aggregate moduli $\overline{\mathrm{H}}_{\mathrm{A}}^{\mathrm{p}}$ and $\overline{\mathrm{H}}_{\mathrm{A}}^{\mathrm{c}}$ are shown in Table 2. The collagen elastic stretch $\lambda_{\mathrm{e}}^{\mathrm{c}}$ (Fig. 3B) and the growth ratio $\mathrm{G}^{\mathrm{p} / \mathrm{c}}$ (Fig. 3C) changed significantly during development $(\mathrm{p}<0.05)$.

CASE A. The results for the collagen material constants $\left(\lambda^{\mathrm{c}}, \mu^{\mathrm{c}}, \Gamma^{\mathrm{c}}\right)$ calculated for each developmental stage are shown in Fig 4; only the parameter $\mu^{\mathrm{c}}$ (Fig. 4B) changed significantly during development $(\mathrm{p}<0.05)$. The collagen material constants for the linearized constitutive equation (18) were calculated; only the parameters $\bar{\mu}^{\mathrm{c}}$ and $\overline{\mathrm{H}}_{\mathrm{A}}^{\mathrm{c}}=\bar{\lambda}^{\mathrm{c}}+2 \bar{\mu}^{\mathrm{c}}$ changed significantly during development $(\mathrm{p}<0.05)$ (Figure 5). Since these results suggest that the collagen remodels during development, the existence of significant linear correlations were investigated. Linear regression analysis revealed that the collagen material constants $\mu^{\mathrm{c}}(\mathrm{p}$ $<0.01), \bar{\mu}^{\mathrm{c}}(\mathrm{p}<0.01)$, and $\overline{\mathrm{H}}_{\mathrm{A}}^{\mathrm{c}}(\mathrm{p}<0.05$; data not shown $)$ were positively correlated with collagen crosslink density $\gamma^{\mathrm{c}}$ (Fig. 6).

CASE B. When using the collagen stress constitutive equation (20), the only model parameters that are different than those obtained for case A are the material constants $\left(\lambda^{\mathrm{c}}, \mu^{\mathrm{c}}, \Gamma^{\mathrm{c}}\right)$ (Table 3). The parameter $\mu^{\mathrm{c}}$ changed significantly between the fetal and newborn stages $(\mathrm{p}<0.05)$ and the parameter $\lambda^{\mathrm{c}}$ changed significantly between the newborn and adult stages $(p<0.05)$.

For both Cases A and B, the predicted solid matrix material constants $\left(\overline{\mathrm{G}}^{\mathrm{s}}, \overline{\mathrm{k}}^{\mathrm{s}}, \overline{\mathrm{E}}^{\mathrm{s}}\right)$ are the same; these parameters changed significantly during development $(\mathrm{p}<0.05)$ and were positively correlated with collagen crosslink density $(\mathrm{p}<0.01$; Fig. 7$)$.

\section{Discussion}

This work illustrates how a mechanical theory of cartilage growth may be used to describe the complex relationship between the evolving compositional and mechanical properties of proteoglycans, collagens, and collagen crosslinks during articular cartilage development. Although the limitations discussed below prevent the current study from quantitatively describing the biomechanics of developmental growth of articular cartilage, the

\footnotetext{
${ }^{9}$ Inequalities for the material constants of the individual constituents are not used because there is no corresponding uniqueness or stability theorem for this material. There is a small-strain uniqueness theorem for a mixture of two elastic solids [47], but here there are constraint responses in the partial stresses and diffusive forces that make that theorem inappropriate.
} 

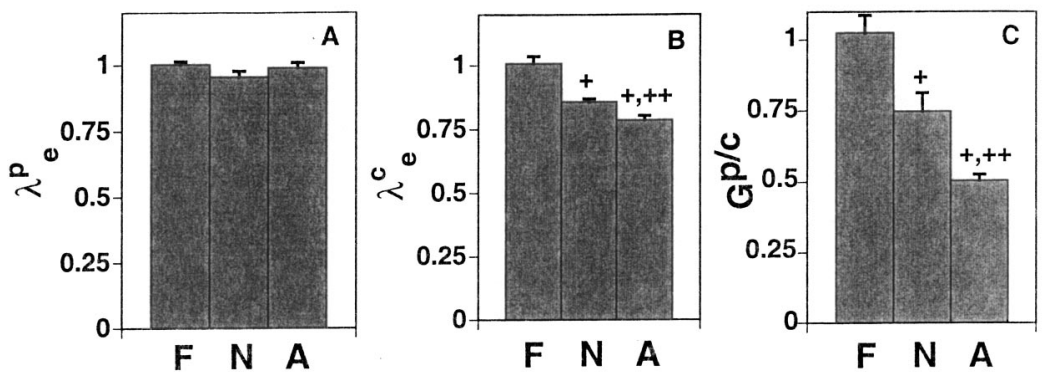

Fig. 3 Model results that describe the kinematics of growth for developing bovine cartilage specimens. Mean \pm S.E.M. results for fetal $(F, n=6)$, newborn $(N, n=8)$, and adult $(A, n=7)$ groups. $(+)$ indicates a significant difference between either $\mathbf{N}$ or $\mathbf{A}$ groups and the $\mathrm{F}$ group, $(++)$ indicates a significant difference between $\mathrm{N}$ and $\mathrm{A}$ groups (ANOVA, Tukey post-hoc, $\mathrm{p}<0.05$ ).

results do offer a qualitative description that may be used to guide future studies. Since different results were obtained for the two assumed collagen stress constitutive equations, it is clear that the use of accurate constitutive equations will be crucial for both validating the model and for using the model to make predictions concerning the biomechanics of cartilage growth. The results are consistent with the experimental observation that the intrinsic properties of the collagen network change during developmental growth to produce a stiffer collagen network [15-17]. The significant correlations between the collagen and solid matrix material constants and the collagen crosslink density suggest as in $[15,17]$ that these changes are partly explained by the increasing crosslink density during development; and indicate that other collagen remodeling parameters (such as collagen content, fiber diameter, and orientation) may be influencing the mechanical properties of the collagen network. Thus, a suggestion for future studies is to obtain additional measures of the collagen microstructure that may evolve during developmental growth.

A limitation of the example presented here is the accuracy of the stress-strain constitutive equations. The development of comprehensive and accurate constitutive equations for use in continuum models of cartilage mechanics has proven to be a formidable challenge for many years. For the cartilage growth mixture model presented here, separate constitutive equations are needed for the proteoglycan and the collagen constituents. In this paper, two simple nonlinear constitutive equations for the collagen stress were used that have not been validated by experimental data. In the cartilage growth example presented in [31], nonlinear stressstrain equations were used for the proteoglycan and collagen con- stituents; in that paper, an exponential collagen stress equation was used that also has not been validated to multiple sets of experimental data. Since the elastic deformations due to growth predicted in the current study are large, it is clear that an immediate aim of future experimental studies should be to develop more accurate nonlinear stress-strain constitutive equations.

One advantage to using a growth theory for large (as opposed to infinitesimal) deformations is that the material properties in the stress constitutive equations, when defined relative to the grown configuration, may evolve during the growth process as the tissue composition changes. To be more specific, when the tissue grows from a reference configuration to a grown configuration, the collagen density may change, which also means that the collagen elastic accommodation tensor will change. When the collagen stress depends on the collagen elastic accommodation tensor nonlinearly, the stress response function relative to the grown configuration will be different than that relative to the reference configuration. In this paper, the manner in which the collagen material constants relative to the grown configuration evolve during isotropic growth were calculated for two assumed collagen stress equations; see Eqs. $(19,22)$. Thus, the cartilage growth mixture model can predict how the solid matrix material constants evolve due to the elastic deformations that are developed to maintain a compatible solid matrix during a growth process. In addition, the model presented here provides a quantitative method for describing how the material constants change due to a remodeling of the collagen constituent.

Several experimental studies have quantified the nonlinear and nonhomogeneous properties of the stress constitutive equations
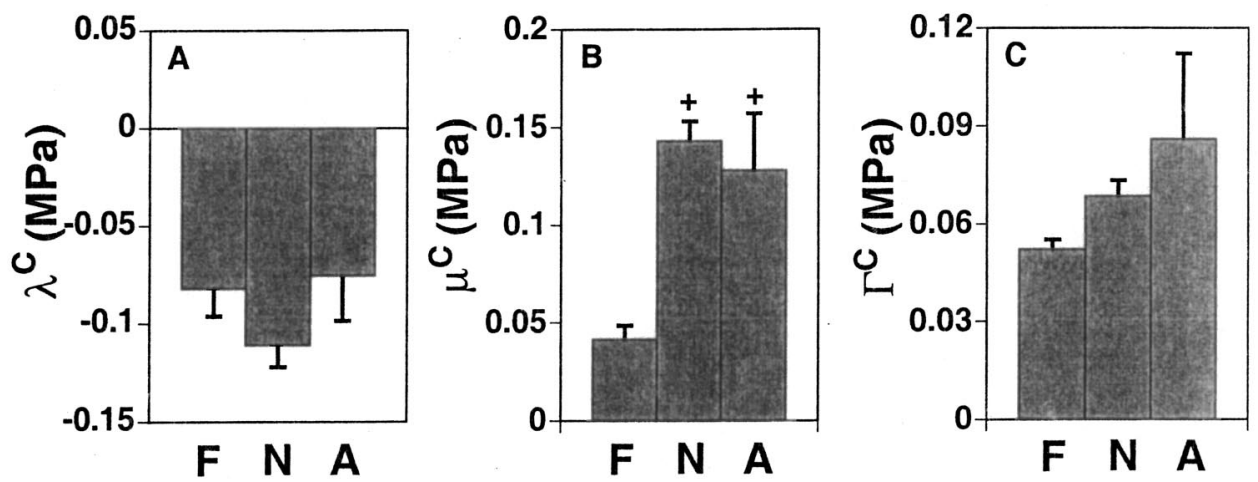

Fig. 4 CASE A model results that describe the evolution of the collagen material constants $\left(\lambda^{c}, \mu^{c}, \Gamma^{c}\right)$ for developing bovine cartilage specimens using the collagen stress equation (16). Mean \pm S.E.M. results for fetal $(F, n=6)$, newborn $(N, n=8)$, and adult $(A, n=7)$ groups. (+) indicates a significant difference between either $\mathrm{N}$ or $\mathrm{A}$ groups and the $\mathrm{F}$ group (ANOVA, Tukey post-hoc, $\mathrm{p}<0.05)$. 

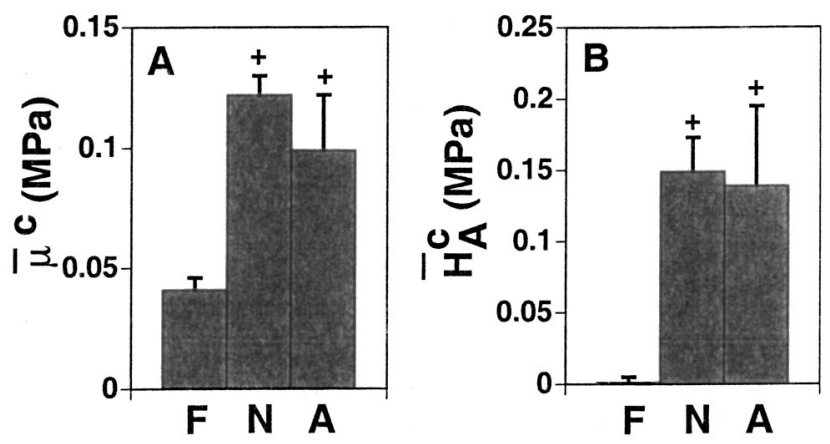

Fig. 5 CASE A model results that describe the evolution of the collagen material constants $\left(\bar{\mu}^{\mathrm{c}}, \bar{H}_{A}^{\mathrm{c}}\right)$ for developing bovine cartilage specimens using the collagen stress equation (18). Mean \pm S.E.M. results for fetal $(F, n=6)$, newborn $(N, n=8)$, and adult $(A, n=7)$ groups. (+) indicates a significant difference between either $\mathbf{N}$ or $\mathbf{A}$ groups and the $\mathbf{F}$ group (ANOVA, Tukey post-hoc, $\mathrm{p}<0.05$ ).

for the cartilage solid matrix. Recently, a model for the solid matrix of cartilage has been proposed [48] that is capable of modeling the tension-compression asymmetry that has been observed in developing cartilage, mature cartilage, and tissue-engineered constructs; and this model was validated for multiple experimental protocols. Also, many studies [49-51] have quantified the depthdependent mechanical properties of bovine and human articular cartilage. However, these nonlinear [48] and nonhomogeneous [49-51] models have only been postulated for infinitesimal strains. In order to develop constitutive equations that may be used accurately during a growth process, they must be validated for multiple experimental protocols and finite strains. Specifically, it will be necessary to conduct multiple mechanical tests such as confined compression, unconfined compression, simple tension and shear in a region-specific manner to more accurately depict the evolving mechanical properties of cartilage between developmental stages. It is emphasized that accurate constitutive equations are necessary in order to predict the evolution of the growth tensors and, consequently, the growth law in the cartilage growth mixture model. In the meantime, the type of study presented in this paper can only be expected to give a qualitative description of the biomechanics of cartilage growth.

Another limiting assumption of the present paper is that the cartilage explants were homogeneous and free of residual stress. In agreement with these assumptions, the growth and elastic accommodation deformations were assumed to be isotropic tensors
Table 3 CASE B mean \pm S.D. values for the collagen material constants $\left(\lambda^{c}, \mu^{c}, \Gamma^{c}\right)$ for the fetal $(F, n=6)$, newborn $(N, n=8)$, and adult $(A, n=7)$ specimens. $(+)$ indicates a significant difference between either $N$ or $A$ groups and the $F$ group. (++) indicates a significant difference between $N$ and $A$ groups (ANOVA, Tukey post-hoc, $\mathrm{p}<0.05$ ).

\begin{tabular}{cccc}
\hline \hline Group & $\begin{array}{c}\lambda^{\mathrm{c}} \\
(\mathrm{MPa})\end{array}$ & $\begin{array}{c}\mu^{\mathrm{c}} \\
(\mathrm{MPa})\end{array}$ & $\begin{array}{c}\Gamma^{\mathrm{c}} \\
(\mathrm{MPa})\end{array}$ \\
\hline $\mathrm{F}$ & -0.027 & -0.013 & -0.053 \\
& $(0.020)$ & $(0.006)$ & $(0.007)$ \\
$\mathrm{N}$ & -0.025 & 0.057 & 0.070 \\
& $(0.016)$ & $(0.042)+$ & $(0.012)$ \\
$\mathrm{A}$ & 0.025 & 0.028 & 0.088 \\
& $(0.061)++$ & $(0.058)$ & $(0.063)$ \\
\hline \hline
\end{tabular}

in the present study. However, there does exist experimental evidence that the deposition of proteoglycan molecules in the extracellular matrix is anisotropic [52] and that the solid matrix may be residually stressed [53]. Thus, another suggestion for future studies will be to conduct experiments that are aimed at quantifying both the orientation of mass deposition and the residual stress field in cartilage explants. It is emphasized that the limitations discussed above are limitations of the assumptions used to obtain a solution for the boundary-value problem; they are not limitations of the cartilage growth mixture model nor the more general growth mixture theory derived in [30,31].

A key feature of cartilage growth is a dependence on mechanical and biochemical stimuli. For example, alterations to the normal in vivo mechanical loading may cause growth abnormalities in conditions such as hip dysplasia [54]. In vivo models such as experimental arthritis and joint immobilization indicate that altered mechanical loads cause remodeling of the compositional and mechanical properties of the solid matrix [55]. In vitro experiments have quantified the solid matrix metabolic response to mechanical stimuli such as hydrostatic pressure, dynamic compressive stress, and fluid diffusion [55]. In these in vitro experiments, the metabolic activity related to proteoglycan and collagen deposition has been measured. The results suggest that the proteoglycan and collagen constituents can grow and remodel independently of each other. Also, the results of numerous studies suggest that biochemical factors influence chondrocyte metabolism. Anabolic agents, such as insulin-like growth factor-1 (IGF-1), transforming growth factor- $\beta$ (TGF- $\beta$ ), and basic fibroblast growth factor (bFGF) stimulate the biosynthesis of proteoglycans and/or collagens [56].

Since the proteoglycan and collagen constituents serve distinct
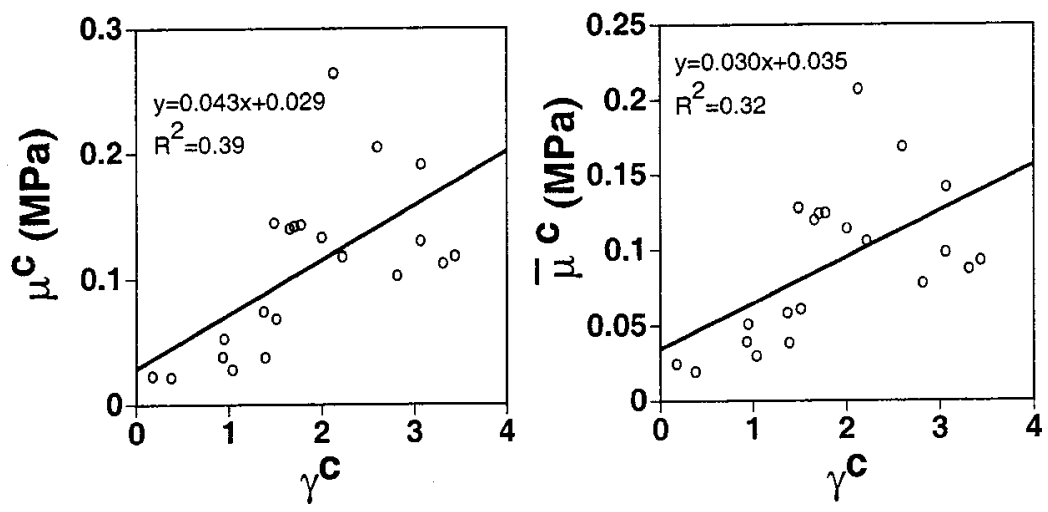

Fig. 6 CASE A linear regression curves for the collagen material constants $\left(\mu^{c}, \bar{\mu}^{c}\right)$ for the F/N/A groups, respectively. Both collagen material parameters were positively correlated $(p<0.01)$ with collagen crosslink density $\gamma^{\mathrm{c}}$ (normalized by the average for the $F$ group). 

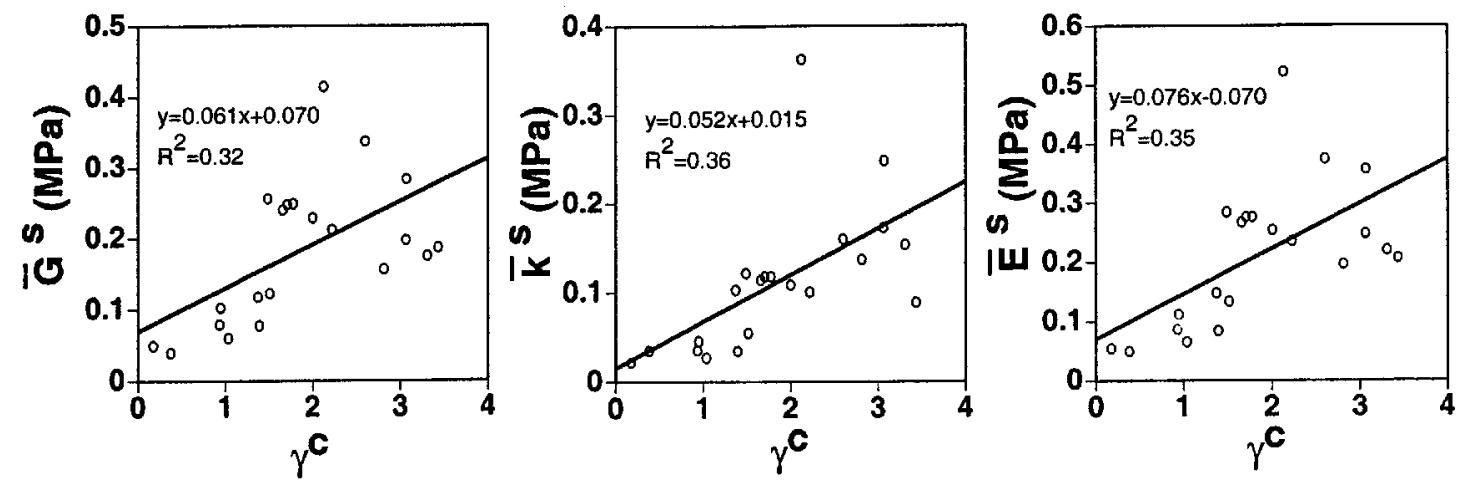

Fig. 7 Linear regression curves for the predicted solid matrix material constants $\left(\bar{G}^{s}, \overline{\boldsymbol{k}}^{\mathrm{s}}, \bar{E}^{\mathrm{s}}\right)$ for the F/N/A groups, respectively. All three solid matrix material parameters were positively correlated $(p<0.01)$ with collagen crosslink density $\gamma^{\mathrm{c}}$ (normalized by the average for the $\mathrm{F}$ group).

mechanical roles, a cartilage growth model should allow for independent constituent growth in order to predict how the mechanical properties of the tissue evolve during growth and degeneration. In a mechanical model of cartilage growth, growth laws such as Eq. $(\mathrm{A} 10)_{1}$ are needed to quantify how the growth of the collagen and proteoglycan constituents depend on mechanical and other stimuli. As a precursor to establishing the forms of these growth laws, in this paper the growth ratio was calculated from measurements of the tissue's change in composition. In order to determine realistic growth laws, the individual components of the growth tensors need to be determined. Experimental data that quantifies the changes in both the geometry of a growing tissue explant as well as the constituent densities would allow one to calculate the determinants of each growth tensor. The type of experiment that was conducted in [52] that quantified the spatial location of molecular deposition in the extracellular matrix could be used to fully characterize each growth tensor. Furthermore, the effect of various biochemical regulators on the growth law may be studied.

The cartilage growth model presented here can be used to design experiments that would lead to the refinement of the model. Specifically, we suggest that experimental studies are needed that apply multiple finite deformations to determine accurate nonlinear stress-strain equations, that measure the amount and orientation of mass deposition in response to mechanical stimuli to determine the growth law, and that better characterize microstructural parameters that may influence these constitutive equations as they evolve during the in vivo growth process. Ultimately, one hopes that these efforts may lead to a model that can be used in a number of ways to develop a better understanding of the key features and mechanisms of cartilage growth. For example, a cartilage growth mixture model may be used to predict the manner in which cartilage constructs may be stimulated in vitro for the fabrication of better implants. Also, it may describe and predict the outcome of therapies aimed at the successful repair of growth and degenerative abnormalities. This can be accomplished by applying the model with simple geometries, such as those corresponding to an in vitro specimen, or with complex geometries, such as those corresponding to a joint's articular surface. In either case, computational models of the in vivo and in vitro growth, degeneration, and repair processes will have to be developed as the governing system of equations are both nonlinear and coupled.

\section{Acknowledgments}

Special thanks to Dr. A. Williamson (UCSD) and Profs. K. Masuda and E.J. Thonar (Rush Medical College) for providing experimental data. Funding received from NIH (SMK, RLS), NSF (AH, RLS) and NASA (RLS).

\section{Appendix}

In this appendix, the governing equations in the theory of growth for a mixture of an arbitrary number of thermoelastic materials and a single fluid are recalled from $[30,31,34]$.

\section{A.i Kinematics.}

Consider a mixture $\mathcal{B}$ of $\nu$ constituents: $\nu-1$ growing elastic materials $\mathcal{C}^{\alpha}(\alpha \in[1, \nu-1])$ and an inviscid fluid $\mathcal{C}^{\nu}$. Let $\kappa_{\mathrm{R}}(\mathcal{B})$ be a fixed reference configuration and $\kappa(\mathcal{B})$ a time-dependent loaded configuration of $\mathcal{B}$ during a continuous growth process. The kinematics for each growing elastic constituent $\mathcal{C}^{\alpha}$ is the same as that adopted for a growing elastic material in [34]. In particular, the deformation gradient tensor for each growing elastic material obeys the decomposition

$$
\mathbf{F}^{\alpha}=\mathbf{M}_{\mathrm{e}}^{\alpha} \mathbf{M}_{\mathrm{g}}^{\alpha}, \quad \alpha \in[1, \nu-1] .
$$

The tensor $\mathbf{M}_{\mathrm{e}}^{\alpha} \mathbf{M}_{\mathrm{g}}^{\alpha}$ describes the total deformation due to growth relative to $\kappa_{\mathrm{R}}(\mathcal{B})$, where $\mathbf{M}_{\mathrm{e}}^{\alpha}$ may include a contribution from a superposed elastic deformation. The amount and orientation of mass deposition are described by $\mathbf{M}_{\mathrm{g}}^{\alpha}$. Furthermore, the mass density, the free energy density, and the stress functions are independent of $\mathbf{M}_{\mathrm{g}}^{\alpha}$.

\section{A.ii Balance Laws.}

In $[30,31]$, the classical balance laws for a mixture were modified to include variables associated with mass deposition and growth energy supply for each growing elastic material. These scalar variables were the mass growth function $\mathrm{c}^{\alpha}$ (i.e., the rate of mass deposition per unit current mass) and the growth energy term $\beta^{\alpha}$ (i.e., the rate of growth energy per unit current mass that is required in addition to that needed to create material with the same internal and kinetic energy as the existing material). The balance equations for mass, linear momentum, angular momentum, and energy on $\kappa(\mathcal{B})$ are

$\frac{\mathrm{d}^{\alpha} \rho^{\alpha}}{\mathrm{dt}}+\rho^{\alpha} \operatorname{div} \mathbf{v}^{\alpha}=\rho^{\alpha} \mathrm{c}^{\alpha}, \quad(\alpha \in[1, \nu-1]) ; \quad \frac{\mathrm{d}^{\nu} \rho^{\nu}}{\mathrm{dt}}+\rho^{\nu} \operatorname{div} \mathbf{v}^{\nu}=0$,

$$
\begin{gathered}
\rho^{\alpha} \frac{\mathrm{d}^{\alpha} \mathbf{v}^{\alpha}}{\mathrm{dt}}=\operatorname{div} \mathbf{T}^{\alpha}+\boldsymbol{\pi}^{\alpha}+\rho^{\alpha} \mathbf{b}^{\alpha}, \\
\mathbf{T}^{\alpha}-\mathbf{T}^{\alpha^{\mathrm{T}}}=\boldsymbol{\Lambda}^{\alpha},
\end{gathered}
$$




$$
\begin{gathered}
\rho^{\alpha} \frac{\mathrm{d}^{\alpha} \varepsilon^{\alpha}}{\mathrm{dt}}=\rho^{\alpha} \mathrm{r}^{\alpha}-\operatorname{div} \mathbf{q}^{\alpha}+\gamma^{\alpha}+\mathbf{T}^{\alpha} \cdot \mathbf{D}^{\alpha}+\rho^{\alpha} \beta^{\alpha}, \quad(\alpha \in[1, \nu-1]) ; \\
\rho^{\nu} \frac{\mathrm{d}^{\nu} \varepsilon^{\nu}}{\mathrm{dt}}=\rho^{\nu} \mathrm{r}^{\nu}-\operatorname{div} \mathbf{q}^{\nu}+\gamma^{\nu}+\mathbf{T}^{\nu} \cdot \mathbf{D}^{\nu},
\end{gathered}
$$

where $\rho^{\alpha}$ is the apparent density, $\mathbf{v}^{\alpha}$ is the velocity, $\mathbf{T}^{\alpha}$ is the partial Cauchy stress tensor, $\boldsymbol{\pi}^{\alpha}$ is the diffusive force, $\mathbf{b}^{\alpha}$ is the partial external body force, $\boldsymbol{\Lambda}^{\alpha}$ is the internal body couple, $\varepsilon^{\alpha}$ is the partial internal energy, $\mathrm{r}^{\alpha}$ is the partial external heat supply, $\mathbf{q}^{\alpha}$ is the partial heat flux vector, $\gamma^{\alpha}$ is the internal energy supply, $\mathbf{D}^{\alpha}$ is the rate of deformation tensor, $\operatorname{div}(\cdot)$ is the divergence operator, and each of the material time derivatives that appear $(A 2-A 5)$ are defined by following a material point of the corresponding constituent. Assuming that the change in density of each growing elastic material $\mathcal{C}^{\alpha}$ is due only to the elastic part of the total deformation of $\mathcal{C}^{\alpha}$, the reduced continuity equation for $\mathcal{C}^{\alpha}$ takes the form

$$
\rho^{\alpha} \operatorname{det} \mathbf{M}_{\mathrm{e}}^{\alpha}=\rho_{\mathrm{R}}^{\alpha}, \quad(\alpha \in[1, \nu-1]),
$$

where $\rho_{\mathrm{R}}^{\alpha}$ is the apparent density in the reference configuration and det $(\cdot)$ is the determinant. Integrating $(A 2)_{1}$ and using $(A 6)$ leads to the growth continuity equation

$$
\operatorname{det} \mathbf{M}_{\mathrm{g}}^{\alpha}=\exp \left\lfloor\int_{\tau=\mathrm{t}_{0}}^{\mathrm{t}} \mathrm{c}^{\alpha} \mathrm{d} \tau\right\rfloor
$$

The balance of mass, linear momentum, and angular momentum equations for the mixture require that

$$
\sum_{\alpha=1}^{\nu-1} \rho^{\alpha} \mathrm{c}^{\alpha}=\rho \mathrm{c}, \quad \sum_{\alpha=1}^{\nu} \boldsymbol{\pi}^{\alpha}=0, \quad \sum_{\alpha=1}^{\nu} \boldsymbol{\Lambda}^{\alpha}=\mathbf{0},
$$

where $\mathrm{c}$ is the mass growth function for the mixture. The balance of energy equation for the mixture becomes

$$
\sum_{\alpha=1}^{\nu}\left[\rho^{\alpha} \frac{\mathrm{d}^{\alpha} \varepsilon^{\alpha}}{\mathrm{dt}}-\rho^{\alpha} \mathrm{r}^{\alpha}+\operatorname{div} \mathbf{q}^{\alpha}+\boldsymbol{\pi}^{\alpha} \cdot \mathbf{v}^{\alpha}-\mathbf{T}^{\alpha} \cdot \mathbf{L}^{\alpha}\right]-\sum_{\alpha=1}^{\nu-1} \rho^{\alpha} \beta^{\alpha}=0 .
$$

\section{A.iii Growth Response Functions.}

To complete the field equations, growth response functions that describe the time-rate of change of $\mathbf{M}_{\mathrm{g}}^{\alpha}$ and $\beta^{\alpha}$ for each growing elastic constituent $\mathcal{C}^{\alpha}$ must be specified. Since the relationship between growth and mechanical stimuli is poorly understood, here growth response functions with the general forms

$$
\frac{\mathrm{d}^{\alpha} \mathbf{M}_{\mathrm{g}}^{\alpha}}{\mathrm{dt}}=\hat{\mathcal{G}}^{\alpha}\left(\mathcal{M}^{\alpha}\right), \quad \beta^{\alpha}=\hat{\beta}^{\alpha}\left(\mathcal{M}^{\alpha}\right)
$$

are considered, where $\mathcal{M}^{\alpha}$ represents the thermomechanical stimuli that drive the growth process for each $\mathcal{C}^{\alpha}$.

\section{A.iv Restrictions Imposed by the Second Law of Thermo-} dynamics.

Constitutive equations are needed for several variables that appear in the theory, including the partial stresses and diffusive forces. In $[30,31]$, this was accomplished by introducing partial free energy and entropy functions and considering restrictions imposed by the Second Law of Thermodynamics after assuming an entropy inequality for the mixtures. The reader is referred to $[30,31]$ for the complete set of constitutive restrictions.

A.v Internal Constraints. Two special types of mechanical constraints that are relevant to cartilage are assumed for the cartilage growth mixture model. One constraint states that the deformation gradient tensors $\mathbf{F}^{\alpha}$ of the growing proteoglycan and collagen constituents are equal. Another constraint is the internal constraint of intrinsic incompressibility [38]. In [30,31], a proce- dure first introduced by Adkins [57] for introducing internal constraints in finite elasticity was used. For a growing mixture subject to a mechanical constraint, the partial stresses and diffusive forces were assumed to obey the additive decompositions

$$
\mathbf{T}^{\alpha}=\widetilde{\mathbf{T}}^{\alpha}+\hat{\mathbf{T}}^{\alpha}, \quad \boldsymbol{\pi}^{\alpha}=\tilde{\boldsymbol{\pi}}^{\alpha}+\hat{\boldsymbol{\pi}}^{\alpha},
$$

where $\left(\widetilde{\mathbf{T}}^{\alpha}, \widetilde{\boldsymbol{\pi}}^{\alpha}\right)$ are indeterminate partial stresses and diffusive forces called the constraint responses, and $\left(\hat{\mathbf{T}}^{\alpha}, \hat{\boldsymbol{\pi}}^{\alpha}\right)$ are determinate partial stresses and diffusive forces. Furthermore, the work done by the constraint response functions was assumed to equal zero.

The requirement that the overall deformation gradient tensors of two growing elastic materials $\mathrm{p}$ and $\mathrm{c}$ be identical leads to the results.

$$
\widetilde{\mathbf{T}}^{\mathrm{p}}=-\widetilde{\mathbf{T}}^{\mathrm{c}}=\tilde{\boldsymbol{\lambda}}, \quad \widetilde{\boldsymbol{\pi}}^{\mathrm{p}}=-\widetilde{\boldsymbol{\pi}}^{\mathrm{c}}=\widetilde{\mathbf{p}},
$$

where $\widetilde{\boldsymbol{\lambda}}$ and $\widetilde{\mathbf{p}}$ are an arbitrary second-order tensor and vector, respectively.

For intrinsic incompressibility, each constituent $\mathcal{C}^{\alpha}$ is assumed to be separable from the others with constant (true) density $\rho^{\alpha T}$ defined as the mass of $\mathcal{C}^{\alpha}$ per unit volume of $\mathcal{C}^{\alpha}$. It is assumed that the volume of the mixture is equal to the sum of the volumes of each $\mathcal{C}^{\alpha}$. In [30,31], the following results for the indeterminate partial stresses and diffusive forces were obtained:

$$
\widetilde{\mathbf{T}}^{\alpha}=\mathrm{p} \frac{\rho^{\alpha}}{\rho^{\alpha \mathrm{T}}} \mathbf{1}, \quad \widetilde{\boldsymbol{\pi}}^{\alpha}=-\mathrm{p} \frac{\operatorname{grad} \rho^{\alpha}}{\rho^{\alpha \mathrm{T}}},
$$

where $\mathrm{p}$ is an arbitrary scalar.

\section{Nomenclature}

$$
\begin{aligned}
& \alpha=\mathrm{p}, \mathrm{c} \text {, w: superscript denoting the proteoglycan, } \\
& \text { collagen, and water constituents } \\
& \kappa_{\mathrm{R}}(\mathcal{B})=\text { fixed reference configuration } \\
& \kappa(\mathcal{B})=\text { grown configuration } \\
& \mathbf{F}^{\alpha}=\text { deformation gradient tensor } \\
& \mathbf{M}_{\mathrm{e}}^{\alpha}=\text { elastic accommodation tensor } \\
& \mathbf{M}_{\mathrm{g}}^{\alpha}=\text { growth tensor } \\
& \rho^{\alpha}=\text { apparent density (mass per tissue volume) } \\
& \rho_{\mathrm{T}}^{\alpha}=\text { true density (mass per constituent volume) } \\
& \operatorname{det}(.)=\operatorname{determinant} \text { of a tensor } \\
& \rho_{\mathrm{R}}^{\alpha}=\text { apparent density in reference configuration } \\
& \mathrm{c}^{\alpha}=\text { mass growth function. } \\
& \operatorname{div}(.)=\text { divergence of a tensor } \\
& \mathbf{T}^{\alpha}=\text { stress tensor } \\
& \mathbf{0}=\text { zero tensor } \\
& \mathbf{T}^{\mathrm{s}}=\text { solid matrix stress tensor } \\
& \widetilde{\mathbf{T}}^{\alpha}=\text { indeterminate part of the stress tensor } \\
& \widetilde{\boldsymbol{\lambda}}=\text { arbitrary second-order tensor } \\
& \mathrm{p}=\text { arbitrary scalar Lagrange multiplier (pore fluid } \\
& \text { pressure) } \\
& \mathbf{1}=\text { identity tensor } \\
& \lambda_{\mathrm{e}}^{\alpha}=\text { elastic stretch } \\
& \mathrm{G}^{\mathrm{p} / \mathrm{c}}=\text { growth ratio } \\
& \hat{\mathbf{T}}^{\alpha}=\text { determinate part of the stress tensor } \\
& \hat{\mathbf{T}}_{\kappa}^{\alpha}=\text { stress constitutive equation relative to the } \\
& \text { grown configuration } \\
& \overline{\mathbf{H}}_{\mathrm{A}}^{\alpha}=\text { aggregate modulus relative to grown configura- } \\
& \text { tion } \\
& \overline{\mathbf{T}}_{\kappa}^{\alpha}=\text { stress constitutive equation for infinitesimal } \\
& \text { elastic strains relative to the grown configura- } \\
& \text { tion } \\
& \operatorname{tr}(.)=\text { trace of a tensor } \\
& \mathbf{e}=\text { infinitesimal solid matrix elastic strain tensor } \\
& \text { superimposed on the grown configuration } \\
& \gamma^{\mathrm{c}}=\text { normalized collagen crosslink density }
\end{aligned}
$$


$\hat{\mathbf{T}}_{\kappa_{\mathrm{R}}}^{\alpha}=$ stress constitutive equation relative to the reference configuration

$\left(\lambda^{\mathrm{c}}, \mu^{\mathrm{c}}, \Gamma^{\mathrm{c}}\right)=$ collagen material constants relative to reference configuration

$\mathbf{R}_{\mathrm{e}}^{\mathrm{c}}=$ rotation tensor associated with $\mathbf{M}_{\mathrm{e}}^{\mathrm{c}}$

$\mathbf{E}_{\mathrm{B}}^{\mathrm{c}}=$ Biot strain tensor

$\mathbf{U}_{\mathrm{e}}^{\mathrm{c}}=$ right stretch tensor associated with $\mathbf{M}_{\mathrm{e}}^{\mathrm{c}}$

$\left(\bar{\lambda}^{\mathrm{c}}, \bar{\mu}^{\mathrm{c}}, \bar{\Gamma}^{\mathrm{c}}\right)=$ collagen material constants relative to grown configuration

$\mathbf{E}^{\mathrm{c}}=$ Lagrangean strain tensor

$\left(\bar{\nu}^{\mathrm{s}}, \bar{G}^{\mathrm{s}}, \bar{k}^{\mathrm{s}}, \bar{E}^{\mathrm{s}}\right)=$ solid matrix material constants relative to grown configuration

\section{References}

[1] Mow, V. C., and Ratcliffe, A., 1997, "Structure and Function of Articular Cartilage and Meniscus," Basic Orthopaedic Biomechanics, ed., V. C. Mow, and W. C. Hayes, Raven Press, New York, pp. 113-178.

[2] Maroudas, A., 1979, "Physico-chemical Properties of Articular Cartilage," Adult Articular Cartilage, ed., M. A. R. Freeman, Pitman Medical, Tunbridge Wells, England, pp. 215-290.

[3] Lai, W. M., Hou, J. S., and Mow, V. C., 1991, "A Triphasic Theory for the Swelling and Deformation Behaviors of Articular Cartilage," J. Biomech. Eng., 113, pp. 245-258.

[4] Basser, P. J., Schneiderman, R., Bank, R., Wachtel, E., et al., 1998, "Mechanical Properties of the Collagen Network in Human Articular Cartilage as Measured by Osmotic Stress Technique," Arch. Biochem. Biophys., 351, pp. $207-$ 219.

[5] Furuto, D. K., and Miller, E. J., 1987, "Isolation and Characterization of Collagens and Procollagens," Methods Enzymol., 144, pp. 41-139.

[6] Eyre, D. R., Koob, T. J., and Van Ness, K. P., 1984, "Quantitation of Hydroxypyridinium Crosslinks in Collagen by High-performance Liquid Chromatography," Anal. Biochem., 137, pp. 380-388.

[7] Woo, S. L.-Y., Akeson, W. H., and Jemmott, G. F., 1976, "Measurements of Nonhomogeneous Directional Mechanical Properties of Articular Cartilage in Tension,” J. Biomech., 9, pp. 785-791.

[8] Venn, M. F., and Maroudas, A., 1977, "Chemical Composition and Swelling of Normal and Osteoarthritic Femoral Head Cartilage I: Chemical Composition,' Annals Rheum. Dis. 36, pp. 121-129.

[9] Sah, R. L., Trippel, S. B., and Grodzinsky, A. J., 1996, "Differential Effects of Serum, IGF-I, and FGF-2 on the Maintenance of Cartilage Physical Properties During Long-term Culture," J. Orthop. Res., 14, pp. 44-52.

[10] Treppo, S., Koepp, H., Quan, E. C., Cole, A. A., et al., 2000, "Comparison of Biomechanical and Biochemical Properties of Cartilage from Human Knee and Ankle Pairs," J. Orthop. Res., 18(5), pp. 739-748.

[11] Maroudas, A., Bullough, P., Swanson, S. A. V., and Freeman, M. A. R., 1968 "The Permeability of Articular Cartilage," J. Bone Jt. Surg., 50-B, pp. 166177.

[12] Pal, S., Tang, L.-H., Choi, H., Habermann, E., et al., 1981, "Structura Changes During Development in Bovine Fetal Epiphyseal Cartilage," Coll. Relat. Res., 1, pp. 151-176.

[13] Strider, W., Pal, S., and Rosenberg, L., 1975, "Comparison of Proteoglycans from Bovine Articular Cartilage," Biochim. Biophys. Acta, 379, pp. 271-281.

[14] Thonar, E. J.-M., and Sweet, M. B. E., 1981, "Maturation-related Changes in Proteoglycans of Fetal Articular Cartilage," Arch. Biochem. Biophys., 208, pp. 535-547.

[15] Chen, S. S., and Sah, R. L., 2001, "Contributions of Collagen Network and Fixed Charge to the Confined Compression Modulus of Articular Cartilage," J. Biomech., (submitted)

[16] Wong, M., Ponticiello, M., Kovanen, V., and Jurvelin, J. S., 2000, "Volumetric Changes of Articular Cartilage During Stress Relaxation in Unconfined Compression," J. Biomech., 33(9), pp. 1049-1054.

[17] Williamson, A. K., Chen, A. C., Masuda, K., Thonar, E. J., et al., 2001, "Evolution of the Biomechanical Properties of Articular Cartilage During Development: Function-composition Relationships," Trans. Annu. Meet. - Orthop. Res. Soc., 26, pp. 427.

[18] Dorland, W. A., 1981, Dorland's illustrated Medical Dictionary, 26th ed., W. B. Saunders Company, Philadelphia.

[19] Fung, Y. C., 1993, Biomechanics: Mechanical Properties of Living Tissues, 2nd ed., Springer-Verlag, New York p. 569.

[20] Skalak, R., Gasgupta, G., Moss, M., Otten, E., et al., 1982, "Analytical Description of Growth," J. Theor. Biol., 94, pp. 555-577.

[21] Taber, L., 1998, "A Model for Aortic Growth Based on Fluid Shear and Fiber Stresses," ASME J. Biomech. Eng., 120, pp. 348-354.

[22] Johnson, B. E., and Hoger, A., 1993, "The Dependence of the Elasticity Tensor on Residual Stress,” J. Elast., 33, pp. 145-165.

[23] Merriam-Webster Collegiate Dictionary, 2001, 10th, http://www.m-w.com/ dictionary.htm

[24] Truesdell, C., and Toupin, R. A., 1960, "The Classical Field Theories," Handbuch der Physik, ed., S. Flugge, Springer-Verlag, Berlin, III/I.

[25] Muller, I., 1968, "A Thermodynamic Theory of Mixtures of Fluids," Arch. Ration. Mech. Anal., 28, pp. 1-39.
[26] Green, A. E., and Naghdi, P. M., 1970, “The Flow of Fluid Through an Elastic Solid," Acta Mech., 9, pp. 329-340.

[27] Bowen, R. M., 1980, "Incompressible Porous Medial Models by use of the Theory of Mixtures," Int. J. Eng. Sci., 18, pp. 1129-1148.

[28] Mow, V. C., Kuei, S. C., Lai, W. M., and Armstrong, C. G., 1980, "Biphasic Creep and Stress Relaxation of Articular Cartilage in Compression: Theory and Experiment," J. Biomech. Eng., 102, pp. 73-84.

[29] Ateshian, G. A., Warden, W. H., Kim, J. J., Grelsamer, R. P., et al., 1997, "Finite Deformation Biphasic Material Properties of Bovine Articular Cartilage from Confined Compression Experiments," J. Biomech., 30, pp. 11571164

[30] Klisch, S. M., and Hoger, A., 2002, "Volumetric Growth of Thermoelastic Materials and Mixtures," Math. Mech. Solids, (in press).

[31] Klisch, S. M., Sah, R. L., and Hoger, A., 2000, "A Growth Mixture Theory for Cartilage," ASME Mechanics in Biology, BED-46, pp. 229-242.

[32] Rodriguez, E. K., Hoger, A., and McCulloch, A. D., 1994, "Stress-dependent Finite Growth in Soft Elastic Tissues," J. Biomech., 27(4), pp. 455-467.

[33] Van Dyke, T. J., and Hoger, A., 2002, "Should the Growth Law be Defined on the Current or Initial Configuration?," J. Theor. Biol., (submitted).

[34] Klisch, S. M., Van Dyke, T, and Hoger, A., 2001, "A Theory of Volumetric Growth for Compressible Elastic Materials,” Math. Mech. Solids, 6, pp. $551-$ 575.

[35] Epstein, M., and Maugin, G. A., 2000, "Thermomechanics of Volumetric Growth in Uniform Bodies,” Int. J. Plast., 16, pp. 951-978.

[36] Humphrey, J. D., and Rajagopal, K. R., 2002, “A Constrained Mixture Model for Growth and Remodeling of Soft Tissues," Mathematical Models and Methods in Applied Science, 12(3), pp. 407-430.

[37] Johnson, B. E., and Hoger, A., 1995, "The Use of a Virtual Configuration in Formulating Constitutive Equations for Residually Stressed Elastic Materials," J. Elast., 41, pp. 177-215.

[38] Mills, N., 1966, "Incompressible Mixtures of Newtonian Fluids," Int. J. Eng. Sci., 4, pp. 97-112

[39] Frank, E. H., and Grodzinsky, A. J., 1987, "Cartilage Electromechanics-II. A Continuum Model of Cartilage Electrokinetics and Correlation with Experiments," J. Biomech., 20, pp. 629-639.

[40] Bachrach, N. B., Mow, V. C., and Guilak, F., 1998, "Incompressibility of the Solid Matrix of Articular Cartilage Under High Hydrostatic Pressures," J. Biomech., 31, pp. 445-451.

[41] Sajdera, S. W., and Hascall, V. C., 1969, "Proteinpolysaccharide Complex from Bovine Nasal Cartilage. A Comparison of Low and High Shear Extraction Procedures," J. Biol. Chem., 244, pp. 77-87.

[42] Pottenger, L. A., Webb, J. E., and Lyon, N. B., 1985, "Kinetics of Extraction of Proteoglycans from Human Cartilage," Arthritis Rheum., 28, pp. 323-330.

[43] Zhu, W., Mow, V. C., Koob, T. J., and Eyre, D. R., 1993, "Viscoelastic Shear Properties of Articular Cartilage and the Effects of Glycosidase Treatment," J. Orthop. Res., 11, pp. 771-781.

[44] Hoger, A., 1999, "A Second Order Constitutive Theory for Hyperelastic Materials," Int. J. Solids Struct., 36, pp. 847-868.

[45] Truesdell, C., and Noll, W., 1965, "The Non-Linear Field Theories of Mechanics," Handbuch der Physik, ed., S. Flugge, Springer-Verlag, Berlin, III/III.

[46] Williamson, A. W., Chen, A. C., and Sah, R. L., 2001, "Compressive Properties and Structure-function Relationships of Developing Bovine Articular Cartilage," J. Orthop. Res., (in press).

[47] Atkin, R. J., Chadwick, P., and Steel, T. R., 1967, "Uniqueness Theorems for Linearized Theories of Interacting Continua," Mathematika, 14, pp. 27-42.

[48] Soltz, M. A., and Ateshian, G. A., 2000, “A Conewise Linear Elasticity Mixture Model for the Analysis of Tension-compression Nonlinearity in Articular Cartilage," ASME J. Biomech. Eng., 122, pp. 576-586.

[49] Schinagl, R. M., Gurskis, D., Chen, A. C., and Sah, R. L., 1997, "Depthdependent Confined Compression Modulus of Full-thickness Bovine Articular Cartilage," J. Orthop. Res., 15, pp. 499-506.

[50] Chen, A. C., Bae, W. C., Schinagl, R. M., and Sah, R. L., 2001, "Depth- and strain-dependent Mechanical and Electromechanical Properties of Fullthickness Bovine Articular Cartilage in Confined Compression," J. Biomech., 34, pp. 1-12.

[51] Chen, S. S., Falcovitz, Y. H., Schneiderman, R., Maroudas, A. et al., 2001, "Depth-dependent Compressive Properties of Normal Aged Human Femoral Head Articular Cartilage," Osteoarthritis Cartilage, (in press)

[52] Buschmann, M. D., Maurer, A. M., Berger, E., and Hunziker, E. B., 1996, "A Method of Quantitative Autoradiography for the Spatial Localization of Proteoglycan Synthesis Rates in Cartilage," J. Histochem. Cytochem., 44, pp. 423-431.

[53] Setton, L. A., Toyama, H., and Mow, V. C., 1998, "Swelling and Curling Behaviors of Articular Cartilage,” ASME J. Biomech. Eng., 120, pp. 355-361.

[54] Pauwels, F., 1976, Biomechanics of the Normal and Diseased Hip; Theoretical Foundations, Technique and Results of Treatment: An Atlas, ed., SpringerVerlag, New York, 276 pp.

[55] Guilak, F., Sah, R. L., and Setton, L. A., 1997, "Physical Regulation of Cartilage Metabolism," Basic Orthopaedic Biomechanics, ed., V. C. Mow, and W. C. Hayes, Raven Press, New York, pp. 179-207.

[56] Trippel, S. B., 1995, "Growth Factor Actions on Articular Cartilage," J. Rheumatology , 43S, pp. 129-132.

[57] Adkins, J. E., 1958, "Dynamic Properties of Resilient Materials: Constitutive Equations,” Philos. Trans. R. Soc. London, 250A, pp. 519-541. 